\title{
CONSERVING THE WRONG VARIABLES IN GAS DYNAMICS: A RIEMANN SOLUTION WITH SINGULAR SHOCKS
}

\author{
BY
}

BARBARA LEE KEYFITZ (Department of Mathematics, The Ohio State University, Columbus, Ohio 43210)

AND

CHARIS TSIKKOU (Department of Mathematics, The Ohio State University, Columbus, Ohio 43210)

\begin{abstract}
We consider a system of two equations derived from isentropic gas dynamics with no classical Riemann solutions. We show existence of unbounded self-similar solutions (singular shocks) of the Dafermos regularization of the system. Our approach is based on the blowing-up approach of geometric singular perturbation theory.
\end{abstract}

1. Introduction. This paper brings together two ideas that have been studied before: the study of large-data solutions to systems of conservation laws, for which wellposedness when the Cauchy data are small in the sense of total variation has been established (see for example [1]), and the study of singular shocks, a type of weak solution of very low regularity, originally described by Keyfitz and Kranzer [11, 12, 13] and later analysed more systematically by Sever [20]. To these constructions, we bring some new ingredients.

Change of type. The system we study is not hyperbolic for all states, but exhibits change of type, with a degeneracy corresponding to vacuum states, and nonhyperbolic states corresponding to negative densities. For some Riemann data, we find the solution includes vacuum states, on the degenerate curve.

A physical model. Whereas the system examined in [11] was derived from a onedimensional model for isothermal, isentropic gas dynamics, a model that is a limit whose correspondence to a genuine physical situation is ambiguous, here we deal with a more standard model, the equations of isentropic gas dynamics for an ideal fluid with $\gamma$, the

Received February 14, 2012.

2010 Mathematics Subject Classification. Primary 35L65, 35L67; Secondary 34E15, 34C37.

Key words and phrases. Conservation laws, singular shocks, Dafermos regularization, geometric singular perturbation theory, nonhyperbolicity, blow-up.

The first author was supported in part by NSF Grant DMS0807569 and by DOE Grant DE-SC0001285.

The second author was supported in part by DOE Grant DE-SC0001285.

E-mail address: bkeyfitz@math.ohio-state.edu

E-mail address: tsikkou@math.ohio-state.edu 
ratio of specific heats, taking any value between 1 and $5 / 3$. As in the isothermal model, we again produce singular shocks by rewriting the equation in a form that conserves different quantities: velocity and entropy. We do not claim that there is any foundation in physics or engineering for this choice, or that the solutions we describe have physical significance or could be demonstrated experimentally. However, we note in passing that a very different model, coming from chromatography, has recently been shown to exhibit singular shocks, and these have indeed been found in carefully designed experiments [15. 16]. This news has motivated us to reexamine the construction of singular shocks and to seek them in different models.

Geometric singular perturbation theory. In addition, since the work of Keyfitz and Kranzer and of Sever, cited above, there has been an interesting development. A derivation of singular shocks as limits of approximations, where the approximations were described only at the level of formal asymptotics, has been improved, using the modern theory of Geometric Singular Perturbation Theory (GSPT) [5, 6] to a theorem that demonstrates the existence of approximate solutions and makes precise their properties. The use of GSPT to analyse singular shocks was pioneered by Schecter [18, using some extensions of Fenichel's theory developed by Krupa and Szmolyan [14] and Schecter and Szmolyan 19. The specific approximation that is amenable to this approach is the socalled Dafermos-DiPerna self-similar regularization of the system. This approximation is not motivated by physics, but has the property that it preserves the self-similar nature of Riemann solutions, which makes it useful here. As the use of GSPT in this context is not yet well known to researchers in conservation laws, we show some details of the extension of Schecter's result, which he proved for the isothermal model, to the wider case we study here. We also correct a small oversight in Schecter's paper.

In the next section, we derive the system of equations we study here: a modification of the isentropic gas dynamics equations that conserves velocity and entropy. In Section 3. we give a formal description of the Riemann solutions, including the cases that include vacuum states. As in [11, we are led to the form of the solutions by using a self-similar viscous perturbation of the system. In Section 4, we summarize Schecter's argument, [18, that proves the existence of self-similar viscous profiles for overcompressive singular shocks for isothermal gas dynamics, and we show that it extends (almost without change) to the isentropic gas dynamics equations (in velocity-entropy form) with $1<\gamma<5 / 3$.

2. Preliminaries. In this section we derive the system we study in this paper and calculate its basic properties (hyperbolicity, genuine nonlinearity, and the shock and rarefaction curves). We identify the region where classical Riemann solutions exist.

2.1. Derivation of the velocity-entropy system from isentropic gas dynamics. We begin with the standard system in Eulerian coordinates:

$$
\left\{\begin{array}{l}
\rho_{t}+\left(u_{1} \rho\right)_{x}=0 \\
\left(\rho u_{1}\right)_{t}+\left(\rho u_{1}^{2}+A \rho^{\gamma}\right)_{x}=0
\end{array}\right.
$$


where $\rho$ is density, $u_{1}$ is velocity, $\gamma>1$ and $A=\frac{\gamma-1}{\gamma}$ (chosen for convenience). With further calculations on the second equation of (2.1.1) we get, using the first equation,

$$
u_{1 t}+\left(\frac{1}{2} u_{1}^{2}+q(\rho)\right)_{x}=0
$$

where $q(\rho)=A \gamma \frac{\rho^{\gamma-1}}{\gamma-1}=\rho^{\gamma-1}$. We write the first equation of (2.1.1) in terms of $u_{1}$ and $q$ using $p(q)=q^{\frac{1}{\gamma-1}}$. We therefore get the following system:

$$
\left\{\begin{array}{l}
u_{1 t}+\left(\frac{1}{2} u_{1}^{2}+q\right)_{x}=0 \\
q_{t}+u_{1} q_{x}+(\gamma-1) u_{1 x} q=0 .
\end{array}\right.
$$

Finally if $\gamma \neq 2$, we use $u_{2}=\frac{2-\gamma}{2} u_{1}^{2}-q$, to derive

$$
\left\{\begin{array}{l}
u_{1 t}+\left(\frac{(3-\gamma)}{2} u_{1}^{2}-u_{2}\right)_{x}=0, \\
u_{2 t}+\left[\frac{(2-\gamma)(5-3 \gamma)}{6} u_{1}^{3}+(\gamma-1) u_{1} u_{2}\right]_{x}=0 .
\end{array}\right.
$$

This system, equivalent to (2.1.1) for smooth solutions, but possessing different weak solutions, expresses conservation of velocity $\left(u_{1}\right)$ and entropy $\left(u_{2}\right)$. We define $U=\left(u_{1}, u_{2}\right)$; the corresponding components in (2.1.4) comprise the flux function $F=F(U)$. We work with the system (2.1.4), with $1<\gamma<\frac{5}{3}$, and Riemann data

$$
U(x, 0)=\left(\begin{array}{l}
u_{1} \\
u_{2}
\end{array}\right)(x, 0)= \begin{cases}U_{L}, & x<0 \\
U_{R}, & x \geq 0 .\end{cases}
$$

For this range of $\gamma$, the system exhibits singular shocks.

2.2. Hyperbolicity and genuine nonlinearity. The Jacobian of (2.1.4) is

$$
\left(\begin{array}{cc}
(3-\gamma) u_{1} & -1 \\
\frac{(2-\gamma)(5-3 \gamma)}{2} u_{1}^{2}+(\gamma-1) u_{2} & (\gamma-1) u_{1}
\end{array}\right) .
$$

The eigenvalues of (2.2.1) are

$$
\begin{aligned}
& \lambda_{1}(U)=u_{1}-\sqrt{\gamma-1} \sqrt{\left(1-\frac{\gamma}{2}\right) u_{1}^{2}-u_{2}}, \\
& \lambda_{2}(U)=u_{1}+\sqrt{\gamma-1} \sqrt{\left(1-\frac{\gamma}{2}\right) u_{1}^{2}-u_{2}} .
\end{aligned}
$$

The eigenvectors are

$$
\begin{aligned}
& r_{1}=\left((2-\gamma) u_{1}+\sqrt{\gamma-1} \sqrt{\left(1-\frac{\gamma}{2}\right) u_{1}^{2}-u_{2}}\right), \\
& r_{2}=\left(\begin{array}{c}
1 \\
\left.(2-\gamma) u_{1}-\sqrt{\gamma-1} \sqrt{\left(1-\frac{\gamma}{2}\right) u_{1}^{2}-u_{2}}\right) .
\end{array} .\right.
\end{aligned}
$$

We denote by $B$ the curve $u_{2}=\left(1-\frac{\gamma}{2}\right) u_{1}^{2}$. The system (2.1.4) is strictly hyperbolic when $u_{2}<\left(1-\frac{\gamma}{2}\right) u_{1}^{2}$, and nonhyperbolic when $u_{2}>\left(1-\frac{\gamma}{2}\right) u_{1}^{2}$. Note that $B$ corresponds to density 0 in the original variables. On $B, \lambda_{1}=\lambda_{2}$ and $r_{1}=r_{2}$.

Since $D \lambda_{i} r_{i}=\frac{\gamma+1}{2}>0$, the states below the curve $B$ are genuinely nonlinear for both $i$-characteristic families. 
2.3. Rarefaction curves through the left state $U_{L}$ in the hyperbolic region. For $i=1$ or 2 , the $i$-rarefaction curves are solutions of the system

$$
\left(\begin{array}{c}
\dot{u}_{1} \\
\dot{u}_{2}
\end{array}\right)=\left((2-\gamma) u_{1} \mp \sqrt{\gamma-1} \sqrt{\left(1-\frac{\gamma}{2}\right) u_{1}^{2}-u_{2}}\right) .
$$

By the change of variables $w=\left(1-\frac{\gamma}{2}\right) u_{1}^{2}$, we get $\frac{d}{d u_{1}}\left(u_{2}-w\right)=\mp \sqrt{\gamma-1} \sqrt{w-u_{2}}$. Upon separation of the variables and integration we have

$$
-2 \sqrt{w-u_{2}}+2 \sqrt{w_{L}-u_{L 2}}=\mp \sqrt{\gamma-1}\left(u_{1}-u_{L 1}\right)
$$

After further calculations we derive the equations for the rarefaction curves through a point $U_{L}$,

$$
\begin{aligned}
R_{1}\left(U_{L}\right): u_{2} & =\frac{(5-3 \gamma)}{4} u_{1}^{2}+u_{1}\left\{\frac{(\gamma-1)}{2} u_{L 1}+\sqrt{\gamma-1} \sqrt{\left(1-\frac{\gamma}{2}\right) u_{L 1}^{2}-u_{L 2}}\right\} \\
& +\left\{-\frac{(3-\gamma)}{4} u_{L 1}^{2}+u_{L 2}-u_{L 1} \sqrt{\gamma-1} \sqrt{\left(1-\frac{\gamma}{2}\right) u_{L 1}^{2}-u_{L 2}}\right\}, \\
R_{2}\left(U_{L}\right): u_{2} & =\frac{(5-3 \gamma)}{4} u_{1}^{2}+u_{1}\left\{\frac{(\gamma-1)}{2} u_{L 1}-\sqrt{\gamma-1} \sqrt{\left(1-\frac{\gamma}{2}\right) u_{L 1}^{2}-u_{L 2}}\right\} \\
& +\left\{-\frac{(3-\gamma)}{4} u_{L 1}^{2}+u_{L 2}+u_{L 1} \sqrt{\gamma-1} \sqrt{\left(1-\frac{\gamma}{2}\right) u_{L 1}^{2}-u_{L 2}}\right\} .
\end{aligned}
$$

The curves $R_{1}$ and $R_{2}$ lie in the closure of the hyperbolic region and intersect only at $U_{L}$. The curves $R_{1}$ and $B$ intersect (tangentially) at

$$
U_{A}=\left(u_{L 1}+\frac{2 \sqrt{\left(1-\frac{\gamma}{2}\right) u_{L 1}^{2}-u_{L 2}}}{\sqrt{\gamma-1}},\left(1-\frac{\gamma}{2}\right)\left[u_{L 1}+\frac{2 \sqrt{\left(1-\frac{\gamma}{2}\right) u_{L 1}^{2}-u_{L 2}}}{\sqrt{\gamma-1}}\right]^{2}\right)
$$

$R_{2}$ and $B$ intersect at

$$
U_{B}=\left(u_{L 1}-\frac{2 \sqrt{\left(1-\frac{\gamma}{2}\right) u_{L 1}^{2}-u_{L 2}}}{\sqrt{\gamma-1}},\left(1-\frac{\gamma}{2}\right)\left[u_{L 1}-\frac{2 \sqrt{\left(1-\frac{\gamma}{2}\right) u_{L 1}^{2}-u_{L 2}}}{\sqrt{\gamma-1}}\right]^{2}\right)
$$

For $1<\gamma<\frac{5}{3}$ and $u_{L 1}>0$, there are four different possibilities for the geometry of $R_{1}$ and $R_{2}$, and when $\frac{3}{2}<\gamma<\frac{5}{3}$ there is one additional case. Similar results hold when $u_{L 1}<0$. These cases are distinguished by the location of the intersection points of $R_{i}$ with the $u_{2}$-axis and with the curve $B$, as well as the minimum and maximum points. In all cases, because $D \lambda_{i} r_{i}=\frac{\gamma+1}{2}>0$, the portion of $R_{i}$ with $u_{1}>u_{L 1}$ corresponds to an admissible rarefaction of the $i$ th family, $i=1$ or 2 . 
2.4. Shock curves through the left state $U_{L}$ in the hyperbolic region. Using the RankineHugoniot jump conditions,

$$
\begin{aligned}
& s\left[u_{1}\right]_{\mathrm{jump}}=\left[\frac{(3-\gamma)}{2} u_{1}^{2}-u_{2}\right]_{\mathrm{jump}}, \\
& s\left[u_{2}\right]_{\mathrm{jump}}=\left[\frac{(2-\gamma)(5-3 \gamma)}{6} u_{1}^{3}+(\gamma-1) u_{1} u_{2}\right]_{\mathrm{jump}},
\end{aligned}
$$

we derive

$$
\begin{aligned}
{\left[u_{2}\right]_{\text {jump }} } & =\frac{\left[u_{1}\right]_{\text {jump }}}{2}\left\{-(\gamma-1) u_{1}+\frac{(3-\gamma)}{2}\left(u_{1}+u_{L 1}\right)\right. \\
& \pm \sqrt{\left.-\frac{(5-3 \gamma)(\gamma+1)}{12}\left[u_{1}\right]_{\text {jump }}^{2}+4(\gamma-1)\left[\left(1-\frac{\gamma}{2}\right) u_{L 1}^{2}-u_{L 2}\right]\right\}} \\
s & =\frac{(3-\gamma)}{2}\left(u_{1}+u_{L 1}\right)-\frac{1}{2}\left\{-(\gamma-1) u_{1}+\frac{(3-\gamma)}{2}\left(u_{1}+u_{L 1}\right)\right. \\
& \pm \sqrt{\left.-\frac{(5-3 \gamma)(\gamma+1)}{12}\left[u_{1}\right]_{\text {jump }}^{2}+4(\gamma-1)\left[\left(1-\frac{\gamma}{2}\right) u_{L 1}^{2}-u_{L 2}\right]\right\}}
\end{aligned}
$$

Here we first observe that when $\gamma<\frac{5}{3}$ the system (2.4.1)-(2.4.2) has real solutions only for

$$
\left|u_{1}-u_{L 1}\right| \leq \sqrt{\frac{48(\gamma-1)}{(5-3 \gamma)(\gamma+1)}\left[\left(1-\frac{\gamma}{2}\right) u_{L 1}^{2}-u_{L 2}\right]} .
$$

The choice of sign for $S_{1}$ and $S_{2}$ is found by calculating

$$
\begin{aligned}
& \left.\frac{d u_{2}}{d u_{1}}\right|_{u_{1}=u_{L 1}}=u_{L 1}(2-\gamma) \pm \sqrt{\gamma-1} \sqrt{\left(1-\frac{\gamma}{2}\right) u_{L 1}^{2}-u_{L 2}}, \\
& \left.\frac{d R_{1}}{d u_{1}}\right|_{u_{1}=u_{L 1}}=u_{L 1}(2-\gamma)+\sqrt{\gamma-1} \sqrt{\left(1-\frac{\gamma}{2}\right) u_{L 1}^{2}-u_{L 2}}, \\
& \left.\frac{d R_{2}}{d u_{1}}\right|_{u_{1}=u_{L 1}}=u_{L 1}(2-\gamma)-\sqrt{\gamma-1} \sqrt{\left(1-\frac{\gamma}{2}\right) u_{L 1}^{2}-u_{L 2}}
\end{aligned}
$$

Since shock and rarefaction curves have second-order contact at $U_{L}$, we conclude that the states that can be connected to $U_{L}$ by a 1 -shock or 2 -shock lie on the curves

$$
\begin{aligned}
S_{1}: u_{2}-u_{L 2} & =\frac{(5-3 \gamma)}{4} u_{1}^{2}+\frac{(\gamma-1)}{2} u_{1} u_{L 1}-\frac{(3-\gamma)}{4} u_{L 1}^{2} \\
& +\frac{\left(u_{1}-u_{L 1}\right)}{2} \sqrt{-\frac{(5-3 \gamma)(\gamma+1)}{12}\left(u_{1}-u_{L 1}\right)^{2}+4(\gamma-1)\left[\left(1-\frac{\gamma}{2}\right) u_{L 1}^{2}-u_{L 2}\right]}
\end{aligned}
$$

or

$$
\begin{aligned}
S_{2}: u_{2}-u_{L 2} & =\frac{(5-3 \gamma)}{4} u_{1}^{2}+\frac{(\gamma-1)}{2} u_{1} u_{L 1}-\frac{(3-\gamma)}{4} u_{L 1}^{2} \\
& -\frac{\left(u_{1}-u_{L 1}\right)}{2} \sqrt{-\frac{(5-3 \gamma)(\gamma+1)}{12}\left(u_{1}-u_{L 1}\right)^{2}+4(\gamma-1)\left[\left(1-\frac{\gamma}{2}\right) u_{L 1}^{2}-u_{L 2}\right]},
\end{aligned}
$$




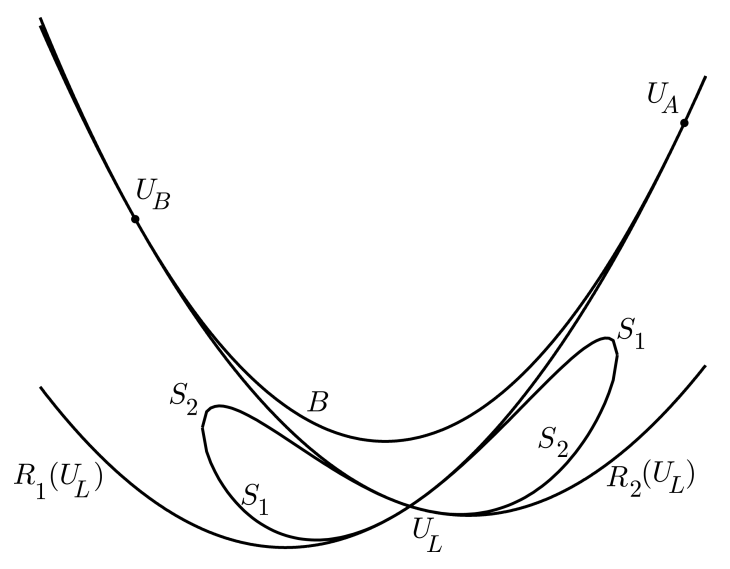

FIG. 2.1. Rarefaction and Shock Curves.

respectively. The curves $S_{1}$ and $S_{2}$ intersect at $U_{L}$ and coincide at points with

$$
u_{1}=u_{L 1} \pm \sqrt{\frac{48(\gamma-1)}{(5-3 \gamma)(\gamma+1)}\left[\left(1-\frac{\gamma}{2}\right) u_{L 1}^{2}-u_{L 2}\right]}
$$

The two shock curves lie between the two (forward and backward) rarefaction curves. The shock curves do not intersect the curve $B$. Figure 2.1 sketches these curves.

2.5. The Lax shock admissibility criterion and classical Riemann solutions. By (2.4.4),

$$
\begin{aligned}
s_{1} & =\frac{(3-\gamma)}{2}\left(u_{1}+u_{L 1}\right)-\frac{1}{2}\left\{-(\gamma-1) u_{1}+\frac{(3-\gamma)}{2}\left(u_{1}+u_{L 1}\right)\right. \\
& +\sqrt{\left.-\frac{(5-3 \gamma)(\gamma+1)}{12}\left(u_{1}-u_{L 1}\right)^{2}+4(\gamma-1)\left[\left(1-\frac{\gamma}{2}\right) u_{L 1}^{2}-u_{L 2}\right]\right\}}, \\
s_{2} & =\frac{(3-\gamma)}{2}\left(u_{1}+u_{L 1}\right)-\frac{1}{2}\left\{-(\gamma-1) u_{1}+\frac{(3-\gamma)}{2}\left(u_{1}+u_{L 1}\right)\right. \\
& -\sqrt{\left.-\frac{(5-3 \gamma)(\gamma+1)}{12}\left(u_{1}-u_{L 1}\right)^{2}+4(\gamma-1)\left[\left(1-\frac{\gamma}{2}\right) u_{L 1}^{2}-u_{L 2}\right]\right\}} .
\end{aligned}
$$

From the eigenvalues (2.2.2)-(2.2.3), we conclude that $\lambda_{1}\left(U_{L}\right)>s_{1}>\lambda_{1}(U)$ and $\lambda_{2}\left(U_{L}\right)$ $>s_{2}>\lambda_{2}(U)$ when $u_{1}<u_{L 1}$. Therefore the admissible parts of the shock curves consist of points with $u_{1}<u_{L 1}$, and the curve of admissible rarefactions has $u_{1}>u_{L 1}$ (if $U_{L}$ is the state on the left).

Using the results of Sections 2.3 and 2.4 and equations (2.5.1)-(2.5.2), we see that in a neighborhood of $U_{L}$ there exist the usual four regions for the solution of the Riemann problem.

Specifically, we have

- Region 1: a 1-shock followed by a 2-shock.

- Region 2: a 1-rarefaction followed by a 2-rarefaction.

- Region 3: a 1-rarefaction followed by a 2-shock.

- Region 4: a 1-shock followed by a 2-rarefaction. 


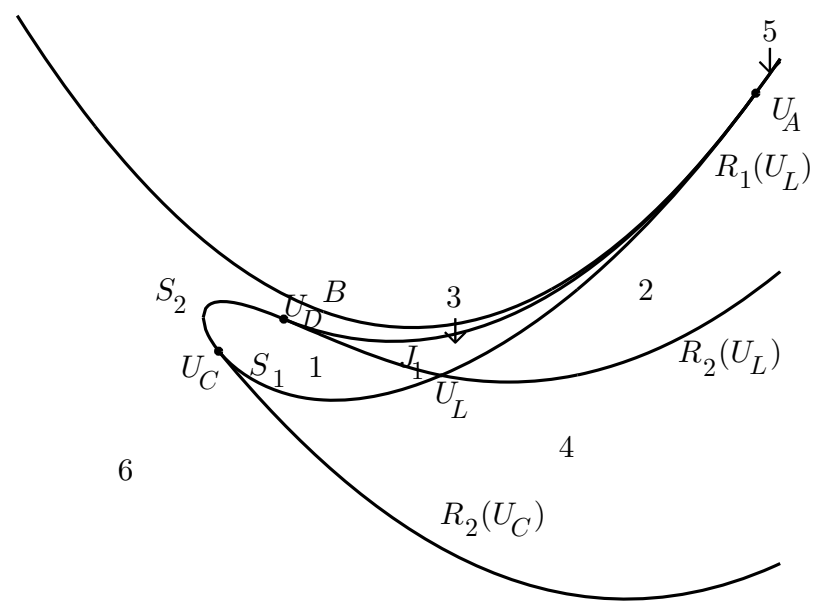

FIG. 2.2. Rarefaction and Shock Curves, Regions.

As a first step in solving the Riemann problem globally we now determine the extent of each of these regions.

Region 1 (two shocks) fills the interior of the loop formed by $S_{1}$ and $S_{2}$ with $u_{1}<u_{L 1}$.

In extending Region 2 to the right, we observe that for any $U_{L}$ and any $\gamma<\frac{5}{3}$ the curve $R_{1}\left(U_{L}\right)$ becomes tangent to $B$ at the point $U_{A}$ identified in equation (2.3.4). The (smooth) continuation of this curve is in fact an $R_{2}$ curve. The fact that $R_{1}(U)$ and $R_{2}(U)$ are almost tangent at points $U$ near the curve $B$ is no surprise. It would have implications for devising estimates for solving Cauchy problems. However with regard to the Riemann problem, we see that a unique solution, consisting of two rarefactions, exists in Region 2, between $R_{2}\left(U_{L}\right)$ and the curve which begins as $R_{1}\left(U_{L}\right)$ and continues as $R_{2}\left(U_{A}\right)$.

Region 3 is, like Region 1, bounded, since only the finite interval of $R_{1}\left(U_{L}\right)$ between $U_{L}$ and $U_{A}$ is available for the intermediate state $U_{M}$. Furthermore, the interval of admissible points $U_{R} \in S_{2}\left(U_{M}\right)$ terminates at a point $U_{D}\left(U_{M}\right)$ at which the shock speed $s_{2}=\lambda_{1}\left(U_{M}\right)$. A calculation shows that the upper boundary of Region 3 is the curve

$$
J_{1}: u_{2}=\left(1-\frac{\gamma}{2}\right) u_{1}^{2}-\frac{(\gamma-1)(5-3 \gamma)}{2(3 \gamma-1)^{2}}\left(u_{1}-u_{L 1}-\frac{2}{\sqrt{\gamma-1}} \sqrt{\left.\left(1-\frac{\gamma}{2}\right) u_{L 1}^{2}-u_{L 2}\right)}\right)^{2} .
$$

This curve is tangent to $S_{2}\left(U_{L}\right)$ at a point $U_{D}$ on $S_{2}\left(U_{L}\right)$, with coordinate

$$
u_{D 1}=u_{L 1}-\sqrt{9(\gamma-1)\left[\left(1-\frac{\gamma}{2}\right) u_{L 1}^{2}-u_{L 2}\right]} .
$$

Finally, Region 4 has as a lower boundary the curve $R_{2}\left(U_{C}\right)$, where $U_{C}$ is the point on $S_{1}\left(U_{L}\right)$ at which $R_{2}\left(U_{C}\right)$ is tangent to $S_{1}\left(U_{L}\right)$; in addition, $s_{1}=\lambda_{2}\left(U_{C}\right)$. The first coordinate of $U_{C}$ is

$$
u_{C 1}=u_{L 1}-\sqrt{\frac{18(\gamma-1)}{(5-3 \gamma)}\left[\left(1-\frac{\gamma}{2}\right) u_{L 1}^{2}-u_{L 2}\right]} .
$$




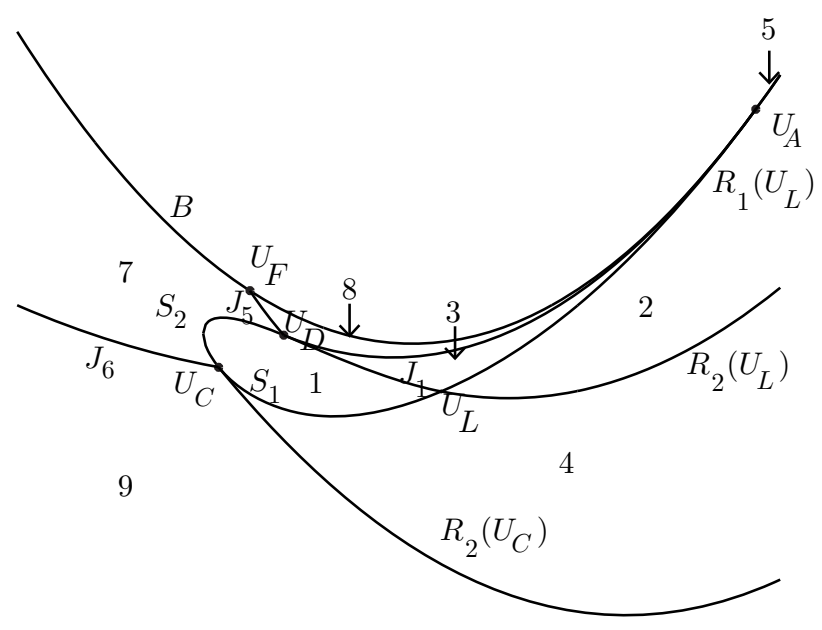

FIG. 2.3. Regions of Singular Shocks, Additional Curves.

2.6. Solutions with a vacuum state. A new type of solution appears in the region labeled Region 5 in Figure 2.2. This region lies between $B$ and the continuation of $R_{1}\left(U_{L}\right)$ as an $R_{2}$ curve beyond the point $U_{A}$. To see how the solution is constructed, we observe that $B$ is an invariant curve for (2.1.4), and if $\left(u_{1}, u_{2}\right)(x, t)$ is a smooth solution on this curve, then $u_{1}$ satisfies the Burgers' equation

$$
u_{1 t}+\left(\frac{u_{1}^{2}}{2}\right)_{x}=0
$$

Thus, if $U_{R}$ is in Region 5, the solution consists of a rarefaction with three separate parts: a 1-rarefaction from $U_{L}$ to $U_{A}$, a rarefaction solution to (2.6.1) from $U_{A}$ to a point $U_{B}\left(U_{R}\right)$, and a 2-rarefaction from $U_{B}\left(U_{R}\right)$ to $U_{R}$; here $U_{B}\left(U_{R}\right)$ is the point where $R_{2}\left(U_{R}\right)$ is tangent to $B$.

Outside these five regions, no classical Riemann solution exists. In the rest of this paper, we show that a solution containing a singular shock can be constructed. Specifically, the Region 6 below $B$, complementary to Regions $1-5$, can be divided into three sets, labeled 7, 8, 9 in Figure 2.3 by curves $J_{5}$ and $J_{6}$, which will be defined in the next section (see equations (3.1.20)-(3.1.21)).

2.7. Remarks. Some additional curves, not needed in the remainder of this paper, are listed here. The points $U$ such that $\lambda_{1}(U)=\lambda_{2}\left(U_{L}\right)$ lie on the curve

$$
J_{2}: u_{2}=\left(1-\frac{\gamma}{2}\right) u_{1}^{2}-\frac{1}{\gamma-1}\left(u_{1}-u_{L 1}-\sqrt{\gamma-1} \sqrt{\left(1-\frac{\gamma}{2}\right) u_{L 1}^{2}-u_{L 2}}\right)^{2} .
$$

The points $U$ such that $\lambda_{1}\left(U_{L}\right)=\lambda_{2}(U)$ lie on the curve

$$
J_{3}: u_{2}=\left(1-\frac{\gamma}{2}\right) u_{1}^{2}-\frac{1}{\gamma-1}\left(u_{1}-u_{L 1}+\sqrt{\gamma-1} \sqrt{\left(1-\frac{\gamma}{2}\right) u_{L 1}^{2}-u_{L 2}}\right)^{2} .
$$


Let $U_{P}$ denote the point such that

$$
s=\frac{\left[\frac{(3-\gamma)}{2} u_{1}^{2}-u_{2}\right]_{\mathrm{jump}}}{\left[u_{1}\right]_{\mathrm{jump}}}=\lambda_{1}\left(U_{L}\right)=\lambda_{2}\left(U_{P}\right),
$$

where $[Y]=Y_{P}-Y_{L}$. We derive that

$$
u_{1 P}=u_{L 1}-2 \sqrt{\gamma-1} \sqrt{\left(1-\frac{\gamma}{2}\right) u_{L 1}^{2}-u_{L 2}} .
$$

Let $U_{M}$ lie on the rarefaction curve $R_{1}\left(U_{L}\right)$ through $U_{L}$, and let $U_{P}\left(U_{M}\right)$ be the point such that

$$
s=\frac{\left[\frac{(3-\gamma)}{2} u_{1}^{2}-u_{2}\right]_{\mathrm{jump}}}{\left[u_{1}\right]_{\mathrm{jump}}}=\lambda_{1}\left(U_{M}\right)=\lambda_{2}\left(U_{P}\left(U_{M}\right)\right),
$$

where $[Y]=Y_{P}\left(Y_{M}\right)-Y_{M}$. We look for the curve of points $U_{P}\left(U_{M}\right)$ as $U_{M}$ moves on $R_{1}\left(U_{L}\right)$ from $U_{L}$ to $U_{A}$. Let $J_{4}$ denote this curve; then

$$
J_{4}: u_{2}=\left(1-\frac{\gamma}{2}\right) u_{1}^{2}-\frac{\gamma-1}{4 \gamma^{2}}\left(u_{1}-u_{L 1}-\frac{2}{\sqrt{\gamma-1}} \sqrt{\left(1-\frac{\gamma}{2}\right) u_{L 1}^{2}-u_{L 2}}\right)^{2} .
$$

Following Sever [21], we conjecture that the points on the curve $J_{4}$ could be used to solve Cauchy problems, with initial data of large oscillation possibly carrying Dirac mass, by the method of a modified front-tracking algorithm.

3. The formal construction of singular shocks. This section begins the construction of singular solutions by examining a self-similar approximation to (2.1.4).

3.1. Dafermos regularization. We study systems that approximate (2.1.4)-(2.1.5). Following Dafermos [2], Dafermos and DiPerna [3], and Keyfitz and Kranzer [11, we approximate a system

$$
U_{t}+F(U)_{x}=0
$$

via Dafermos regularization:

$$
\varepsilon t U_{x x}=U_{t}+F(U)_{x} .
$$

Using $\xi=\frac{x}{t}$, the initial value problem (3.1.1)-(2.1.5) becomes a nonautonomous secondorder ODE

with boundary conditions

$$
\varepsilon \frac{d^{2} U}{d \xi^{2}}=(D F(U)-\xi I) \frac{d U}{d \xi}
$$

$$
U(-\infty)=U_{L}, \quad U(+\infty)=U_{R}
$$

Since in the region of interest there are no classical solutions, we seek solutions that are not uniformly bounded in $\varepsilon$ for $\xi$ near some value $s$. The following technique, first used by Keyfitz and Kranzer [11, provides a formal solution. We develop this and then show in Section 4, following Schecter [18, that for sufficiently small $\varepsilon>0$, (3.1.2) possesses solutions with the qualitative behavior we predict in this section. Although the GSPT analysis should not (and does not) depend on the singular perturbation formalism of this section, the formal calculations are a valuable guide. 
Let

$$
U(\xi)=\left(\begin{array}{c}
\frac{1}{\varepsilon^{p}} \tilde{u}_{1}\left(\frac{\xi-s}{\varepsilon^{q}}\right) \\
\frac{1}{\varepsilon^{r}} \tilde{u}_{2}\left(\frac{\xi-s}{\varepsilon^{q}}\right)
\end{array}\right),
$$

and define $\eta=\frac{\xi-s}{\varepsilon^{q}}$. Then (3.1.2) becomes

$$
\left\{\begin{aligned}
\varepsilon^{1-p-q} \frac{d^{2} \tilde{u}_{1}}{d \eta^{2}}= & \left((3-\gamma) \varepsilon^{-p} \tilde{u}_{1}-\varepsilon^{q} \eta-s\right) \varepsilon^{-p} \frac{d \tilde{u}_{1}}{d \eta}-\varepsilon^{-r} \frac{d \tilde{u_{2}}}{d \eta} \\
\varepsilon^{1-r-q} \frac{d^{2} \tilde{u}_{2}}{d \eta^{2}}= & \left(\frac{(2-\gamma)(5-3 \gamma)}{2} \varepsilon^{-2 p} \tilde{u}_{1}^{2}+(\gamma-1) \varepsilon^{-r} \tilde{u}_{2}\right) \varepsilon^{-p} \frac{d \tilde{u}_{1}}{d \eta} \\
& +\left((\gamma-1) \varepsilon^{-p} \tilde{u}_{1}-\varepsilon^{q} \eta-s\right) \varepsilon^{-r} \frac{d \tilde{u_{2}}}{d \eta}
\end{aligned}\right.
$$

We balance at least two terms in each equation, so that nontrivial solutions can be found. Thus we set $1-p-q=-r$ in the first equation, and either $1-r-q=-p-r$ or $1-r-q=-3 p$ in the second. This gives $r=2 p, q=1+p$ and hence

$$
\left\{\begin{array}{l}
\frac{d^{2} \tilde{u}_{1}}{d \eta^{2}}=(3-\gamma) \tilde{u}_{1} \frac{d \tilde{u}_{1}}{d \eta}-\varepsilon^{p}\left(\varepsilon^{p+1} \eta+s\right) \frac{d \tilde{u}_{1}}{d \eta}-\frac{d \tilde{u_{2}}}{d \eta}, \\
\frac{d^{2} \tilde{u}_{2}}{d \eta^{2}}=\left(\frac{(2-\gamma)(5-3 \gamma)}{2} \tilde{u}_{1}^{2}+(\gamma-1) \tilde{u}_{2}\right) \frac{d \tilde{u}_{1}}{d \eta}+(\gamma-1) \tilde{u}_{1} \frac{d \tilde{u_{2}}}{d \eta}-\varepsilon^{p}\left(\varepsilon^{p+1} \eta+s\right) \frac{d \tilde{u_{2}}}{d \eta} .
\end{array}\right.
$$

We can conclude that none of $r, p$, or $q$ can be zero. In particular, we must have $q>1$, so the singular region is narrower than a standard shock profile.

When we expand $\tilde{u}_{1}, \tilde{u}_{2}$ as series in $\varepsilon$,

$$
\tilde{u}_{1}=\tilde{u}_{10}(\eta)+o(1), \quad \tilde{u}_{2}=\tilde{u}_{20}(\eta)+o(1)
$$

we obtain

$$
\left\{\begin{array}{l}
\frac{d^{2} \tilde{u}_{10}}{d \eta^{2}}=(3-\gamma) \tilde{u}_{10} \frac{d \tilde{u}_{10}}{d \eta}-\frac{d \tilde{u_{0}}}{d \eta} \\
\frac{d^{2} \tilde{u}_{20}}{d \eta^{2}}=\left(\frac{(2-\gamma)(5-3 \gamma)}{2} \tilde{u}_{10}^{2}+(\gamma-1) \tilde{u}_{20}\right) \frac{d \tilde{u}_{10}}{d \eta}+(\gamma-1) \tilde{u}_{10} \frac{d \tilde{u}_{20}}{d \eta} .
\end{array}\right.
$$

Assuming that the singular behavior is restricted to a neighborhood of $\xi=s$, we have

$$
\tilde{u}_{i 0}( \pm \infty)=0, \quad \frac{d \tilde{u}_{i 0}}{d \eta}( \pm \infty)=0, \text { for } i=1,2 .
$$

We integrate (3.1.7) once, simplify notation using $\tilde{u}_{10}=y_{1}, \tilde{u}_{20}=y_{2}$, and now focus attention on solutions of

$$
\left\{\begin{array}{l}
\frac{d y_{1}}{d \eta}=(3-\gamma) \frac{y_{1}^{2}}{2}-y_{2} \\
\frac{d y_{2}}{d \eta}=\frac{(2-\gamma)(5-3 \gamma)}{6} y_{1}^{3}+(\gamma-1) y_{1} y_{2}
\end{array}\right.
$$

which approach $(0,0)$ as $|\eta| \rightarrow \infty$. We will let $Y$ denote $\left(y_{1}, y_{2}\right)$. We note that the vector field in (3.1.8) is $F(Y)$, where $F$ is the flux function in (2.1.4). Now, system (3.1.8) 


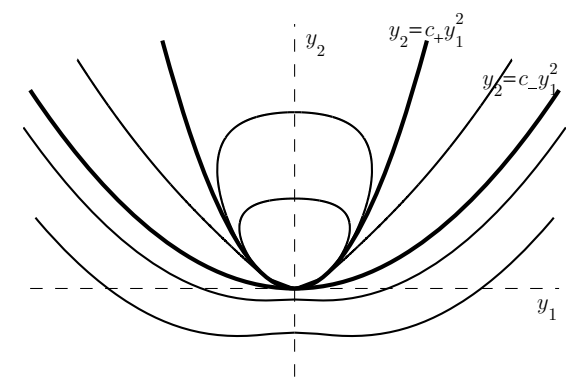

FIG. 3.1. Integral Curves and Orbits of (3.1.8.

admits a first integral

$$
\begin{aligned}
C\left|y_{2}-\left\{1-\frac{\gamma}{2}-\sqrt{\frac{1}{6}\left(1-\frac{\gamma}{2}\right)}\right\} y_{1}^{2}\right| \frac{1}{4}+\frac{1}{8 \sqrt{\frac{1}{6}\left(1-\frac{\gamma}{2}\right)}} \\
=\left|y_{2}-\left\{1-\frac{\gamma}{2}+\sqrt{\frac{1}{6}\left(1-\frac{\gamma}{2}\right)}\right\} y_{1}^{2}\right|^{-\frac{1}{4}+\frac{1}{8 \sqrt{\frac{1}{6}\left(1-\frac{\gamma}{2}\right)}}},
\end{aligned}
$$

where $C$ is a constant. This can be calculated by the substitution $y_{2} \mapsto \theta=y_{2} / y_{1}^{2}$.

The phase portrait of the two-dimensional system (3.1.8) is shown in Figure 3.1. The origin is the unique equilibrium. For $C=0$ and $C=\infty$ we have two invariant parabolas $y_{2}=c_{ \pm} y_{1}^{2}$ with $c_{ \pm}=\left(1-\frac{\gamma}{2}\right) \pm \sqrt{\frac{1}{6}\left(1-\frac{\gamma}{2}\right)}$. The homoclinic orbits, which are of greatest interest to us, are solutions $\left(y_{1}(\eta), y_{2}(\eta)\right)$ which can be determined uniquely by setting $y_{1}(0)=0, y_{2}(0)>0$. We find that $y_{2}(\eta)$ is integrable. (The argument is a minor variant of the proofs presented in [11] and [17].) Each orbit defines a unique value of $k=\int_{-\infty}^{\infty} y_{2}(\eta) d \eta, 0<k<\infty$. Note that $y_{1}(\eta)$ is an odd function and $y_{2}(\eta)$ even and nonnegative. We will need to know the asymptotic behavior of $Y$ as $|\eta| \rightarrow \infty$. Writing

$$
\begin{aligned}
& y_{1}=\frac{m}{\eta}+\mathcal{O}\left(\frac{1}{\eta^{2}}\right), \\
& y_{2}=\frac{n}{\eta^{2}}+\mathcal{O}\left(\frac{1}{\eta^{3}}\right),
\end{aligned}
$$

we substitute (3.1.10)-3.1.11) into (3.1.8) and then solve for $m$ and $n$, to obtain

$$
m=-\frac{6}{\gamma+1} \pm \frac{2 \sqrt{3(2-\gamma)}}{\gamma+1}, \quad n=\frac{(3-\gamma)}{2} m^{2}+m .
$$

We also have

$$
y_{2} \approx-\frac{(2-\gamma)(5-3 \gamma) m}{6[2+m(\gamma-1)]} y_{1}^{2}
$$

as $Y \rightarrow 0$. Using the calculated values of $m$, we obtain the two parabolas

$$
y_{2}=d_{ \pm} y_{1}^{2}
$$


where

$$
d_{ \pm}=\frac{(5-3 \gamma)}{6}+\frac{(5-3 \gamma)}{6} \frac{(\sqrt{2-\gamma} \pm \sqrt{3})}{(2 \sqrt{2-\gamma} \mp \sqrt{3}(\gamma-1))}
$$

These describe the asymptotic behavior of $Y$ as $Y \rightarrow 0$. We observe that $\frac{3-\gamma}{2}>c_{+}=$ $d_{+}>c_{-}=d_{-}$. Therefore the homoclinic orbits are tangent to the invariant parabola $y_{2}=c_{+} y_{1}^{2}$ at both ends.

The singular solution (3.1.4) has its essential support in a layer of width $|\xi-s|=O\left(\varepsilon^{q}\right)$ with $q>1$ and tends to zero away from $\xi=s$. As in Keyfitz and Kranzer [11 we embed the singular shock in a shock profile of the usual type: a solution $\bar{U}(\tau)=\bar{U}\left(\frac{\xi-s}{\varepsilon}\right)$, which is bounded in the layer $\varepsilon^{q}<|\xi-s|<\varepsilon$, has an expansion

$$
\bar{U}=\bar{U}_{0}+o(1)
$$

and whose derivatives are $O\left(\varepsilon^{-1}\right)$.

Writing (3.1.2) in terms of $\tau=\frac{\xi-s}{\varepsilon}$ we have

$$
\frac{d}{d \tau}\left(\frac{d \bar{U}}{d \tau}-F(\bar{U})+s \bar{U}\right)=-\varepsilon \tau \frac{d \bar{U}}{d \tau} .
$$

Using the expansion (3.1.12) we have

$$
\frac{d}{d \tau}\left(\frac{d \bar{U}_{0}}{d \tau}-F\left(\bar{U}_{0}\right)+s \bar{U}_{0}\right)=0
$$

in each separate interval $\tau<0$ or $\tau>0$ outside the boundary layer. Hence, we may write

$$
\frac{d \bar{U}_{0}}{d \tau}-F\left(\bar{U}_{0}\right)+s \bar{U}_{0}=k_{\mp} .
$$

On the other hand, integrating (3.1.13) over a short interval surrounding $\tau=0$ (the boundary layer), we obtain

$$
\left[\frac{d \bar{U}}{d \tau}-F(\bar{U})+s \bar{U}\right]_{\tau<0}^{\tau>0}=-\varepsilon \int_{\tau<0}^{\tau>0} \tau \frac{d \bar{U}}{d \tau} d \tau
$$

Now, from (3.1.4),

$$
\bar{U}(\tau)=\left(\begin{array}{c}
\frac{1}{\varepsilon^{p}} \tilde{u}_{1}(\eta) \\
\frac{1}{\varepsilon^{2 p}} \tilde{u}_{2}(\eta)
\end{array}\right),
$$

and we change the variable to $\eta=\tau / \varepsilon^{p}$ in (3.1.16), which yields

$$
k_{+}-k_{-}=\lim _{\varepsilon \rightarrow 0}\left\{-\varepsilon \int \varepsilon^{p} \eta\left(\begin{array}{c}
\frac{1}{\varepsilon^{p}} \frac{d \tilde{u}_{1}}{d \eta} \\
\frac{1}{\varepsilon^{2 p}} \frac{d \tilde{u}_{2}}{d \eta}
\end{array}\right) d \eta\right\}=\lim _{\varepsilon \rightarrow 0}\left(\begin{array}{c}
-\varepsilon \int \eta \frac{d \tilde{u}_{10}}{d \eta} d \eta \\
-\varepsilon^{1-p} \int \eta \frac{d \tilde{u}_{20}}{d \eta} d \eta
\end{array}\right) .
$$

By (3.1.10)-(3.1.11),

$$
\frac{d \tilde{u}_{10}}{d \eta} \approx-\frac{m}{\eta^{2}}, \frac{d \tilde{u}_{20}}{d \eta} \approx-2 \frac{n}{\eta^{3}}
$$


when $|\eta| \rightarrow \infty$. From the symmetry of the equation and the initial condition $\tilde{u}_{10}(0)=0$, we see that $\eta \frac{d \tilde{u}_{10}}{d \eta}$ is an odd function. It is not absolutely integrable, but has zero integral in the principal value sense. On the other hand,

$$
\int \eta \frac{d \tilde{u}_{20}}{d \eta} d \eta=-\int \tilde{u}_{20} d \eta
$$

exists and has a different value for each homoclinic trajectory. If $p=1$, then

$$
k_{+}-k_{-}=\left(\begin{array}{c}
0 \\
\int \tilde{u}_{20} d \eta
\end{array}\right)=\left(\begin{array}{l}
0 \\
k
\end{array}\right) .
$$

By (3.1.3), for the shock in the solution $\bar{U}$ we have $\bar{U}_{0}(-\infty)=U_{L}, \bar{U}_{0}(+\infty)=U_{R}$ and $\frac{d \bar{U}_{0}}{d \tau}( \pm \infty)=0$. Finally, from (3.1.15) we get the generalized Rankine-Hugoniot condition for singular shocks:

$$
\begin{aligned}
s_{\text {singular }}\left(U_{L}, U_{R}\right) & =s=\frac{F_{1}\left(U_{L}\right)-F_{1}\left(U_{R}\right)}{u_{L 1}-u_{R 1}}, \\
0<k & =F_{2}\left(U_{L}\right)-F_{2}\left(U_{R}\right)-s\left(u_{L 2}-u_{R 2}\right) .
\end{aligned}
$$

(The sign of $k$ is determined by $n>0$ in (3.1.11).) The restriction on the sign of $k$ is consistent with having $U_{R}$ in region 6 with respect to $U_{L}$. Let us divide this region into three parts: regions 7,8 and 9 as shown in Figure 2.3. The boundaries of region 7 are the curves $J_{5}$ and $J_{6}$ determined by

$$
s_{\text {singular }}\left(U_{L}, U\right)=\lambda_{1}\left(U_{L}\right)
$$

and

$$
s_{\text {singular }}\left(U_{L}, U\right)=\lambda_{2}(U)
$$

respectively. We find

$$
\begin{aligned}
J_{5}: u_{2} & =\frac{(3-\gamma)}{2} u_{1}^{2}+u_{1}\left\{\sqrt{\gamma-1} \sqrt{\left(1-\frac{\gamma}{2}\right) u_{L 1}^{2}-u_{L 2}}-u_{L 1}\right\}+u_{L 2}+\frac{(\gamma-1)}{2} u_{L 1}^{2} \\
& -u_{L 1} \sqrt{\gamma-1} \sqrt{\left(1-\frac{\gamma}{2}\right) u_{L 1}^{2}-u_{L 2}} .
\end{aligned}
$$

The curve $J_{5}$ passes through the point $U_{D}$ and intersects $B$ at a point $U_{F}$ with

$$
u_{F 1}=u_{L 1}-(\sqrt{\gamma-1}+\sqrt{\gamma+1}) \sqrt{\left(1-\frac{\gamma}{2}\right) u_{L 1}^{2}-u_{L 2}} .
$$

We find that $u_{B 1}<u_{F 1}$, where $U_{B}=U_{B}\left(U_{L}\right)$ is given by (2.3.5). The second boundary is $J_{6}$, given by

$$
\begin{aligned}
J_{6}: u_{2} & =u_{L 2}+\left(u_{1}-u_{L 1}\right)\left(u_{L 1}-(\gamma-1) u_{1}\right) \\
& -\left(u_{1}-u_{L 1}\right) \sqrt{\frac{\left(\gamma^{2}-1\right)}{4}\left(u_{1}-u_{L 1}\right)^{2}+(\gamma-1)\left[\left(1-\frac{\gamma}{2}\right) u_{L 1}^{2}-u_{L 2}\right] .}
\end{aligned}
$$

The curve $J_{6}$ passes through the point $U_{C}$ and does not intersect $B$.

In the remainder of this paper we show existence of self-similar singular shock solutions to (3.1.1). Our main result is the following theorem. 
TheOREM 3.1. In the system of conservation laws (2.1.4) with Riemann data (2.1.5), and $1<\gamma<5 / 3$, assume that $U_{R}$ is in the interior of region 7 with respect to $U_{L}$, so that with

$$
s_{\text {singular }}\left(U_{L}, U_{R}\right) \equiv \frac{F_{1}\left(U_{L}\right)-F_{2}\left(U_{R}\right)}{u_{L 1}-u_{R 1}},
$$

we have

$$
0<k=F_{2}\left(U_{L}\right)-F_{2}\left(U_{R}\right)-s_{\text {singular }}\left(u_{L 2}-u_{R 2}\right),
$$

and the strict inequalities

(1) $s_{\text {singular }}\left(U_{L}, U_{R}\right)<\lambda_{1}\left(U_{L}\right)$,

(2) $\lambda_{2}\left(U_{R}\right)<s_{\text {singular }}\left(U_{L}, U_{R}\right)$

hold. Then there exists a singular shock connecting $U_{L}$ and $U_{R}$, that is, a solution $U_{\varepsilon}$ of (3.1.2)-(3.1.3) which becomes unbounded as $\varepsilon \rightarrow 0$.

3.2. Remarks. Proving existence of a self-similar approximate solution in region 7 , in which the Riemann problem is solved by a singular shock alone, provides a partial answer to the question of existence of the singular solutions constructed formally in this section. A unique solution, consisting of shocks, rarefactions and singular shocks, is obtained (formally) if we complete the list of solutions in regions 1-5, given in Sections 2.5] and 2.6. with the following constructions:

- region 7, a singular shock, which is strictly overcompressive;

- region 8, a 1-rarefaction to a state $U_{M}$ followed by a singular shock with

$$
s_{\text {singular }}=\lambda_{1}\left(U_{M}\right)
$$

- region 9 , a singular shock to a state $U_{M}$ followed by a 2-rarefaction with

$$
s_{\text {singular }}=\lambda_{2}\left(U_{M}\right) .
$$

In Section 4, we prove Theorem 3.1 by showing the existence of solutions to equations (3.1.2) and (3.1.3) for small $\varepsilon$. We use the approach of Schecter [18, which proceeds by modifying GSP theory [5, 6] to take into account that normal hyperbolicity fails in parts of the construction (as will be detailed below). A method for handling loss of normal hyperbolicity, known as "blowing up", was developed by Krupa and Szmolyan, 14], and applied by Schecter to the $\gamma=1$ case of this problem (which does not have a nonhyperbolic region). Strict overcompressibility is needed, as will be seen, to carry out the construction. In regions 8 or 9 , where the singular shock is merely weakly overcompressive ( $s_{\text {singular }}$ is equal to one of the characteristic speeds), further degeneracies occur. It is possible that GSP theory could be extended to cover this case, but we have not carried out this step. If a singular wave is constructed for states $U_{L}, U_{R}$ with $U_{R}$ outside region 6 , then we conjecture that an attempt to find a self-similar viscous profile would fail. We have not carried out the details of this proof, either.

4. Existence of approximations to singular solutions. We prove Theorem 3.1 by using GSPT to show that self-similar regularized solutions exist for sufficiently small $\varepsilon>0$. The approach was laid out by Schecter [18] for the case $\gamma=1$, and the construction is exactly the same in this case. We give an outline that emphasizes the ideas that might not be familiar to researchers in conservation laws. 
In essence, the situation described in Section 3 is of a formal solution consisting of an "outer" part (which happens, for this example, to be simply the two constant states $U_{L}$ and $U_{R}$ ) and an "inner" part (the scaled homoclinic orbit) with no obvious way to connect the two parts. The classical method of matched asymptotic expansions provides an answer which suffers from the defect of not proving that a solution exists; matched expansions simply suggest a mechanism whereby the two parts of the solution could be connected. Our treatment following (3.1.12) was in this spirit. Geometric singular perturbation theory (GSPT) corrects this, using the theory of dynamical systems to prove that smooth systems, under the appropriate nondegeneracy conditions, do possess connecting orbits, and even that these orbits are unique. GSPT was developed by Fenichel [5] (see also the exposition by Jones [6]), and it cannot be applied directly to this problem; as a central hypothesis, normal hyperbolicity, does not hold. (A flow is normally hyperbolic with respect to an invariant manifold if any manifold transverse to the flow can be factored into stable and unstable directions. More precisely, if the system is linearized at a point on the invariant manifold, then only the eigenvalues with eigenvectors tangent to the invariant manifold have zero real part.) When we write down the system, it will be seen that this condition fails. Krupa and Szmolyan [14] applied their technique of "blowing up" to some examples, but it was Schecter who showed how it could also apply to the system (3.1.2). The insight of GSPT is that one can produce a sort of cartoon of the orbit, a "stick drawing" when $\varepsilon=0$, and then flesh it out by proving the existence of a genuine orbit when $\varepsilon>0$. The homoclinic orbit produced in the previous section is a part of the cartoon, but there was no indication in the construction that produced it of how to tie it to a skeleton that joins $U_{L}$ and $U_{R}$. Within the framework of GSPT, we find, following Schecter, a method for doing so. The second part of this paper is the application of dynamical systems theory to prove the existence of an orbit when $\varepsilon>0$. The important tool here is the Exchange Lemma of Jones and Kopell [8], and an extension called by Schecter [18 the Corner Lemma.

The novelty of the GSPT approach is that it replaces a dynamical problem, here (3.1.2) and (3.1.3), in which a singular limit occurs, with a higher-dimensional dynamical system in which $\{\varepsilon=0\}$ is merely a subspace, and behavior near that subspace can be determined by continuity if the hypotheses of the Exchange and Corner Lemmas are satisfied. In the problem at hand, the interesting dynamics when $\varepsilon=0$ is typically planar. As could already be seen in Section 3.1, some rescaling of the variables is needed to exhibit any of the dynamics on the fast time scale. Thus, we expect to see scale changes. However, if this is done in the standard way, then as we shall see, the invariant subspaces $\{\varepsilon=0\}$ are not normally hyperbolic. This difficulty is surmounted by the technique of "blowing up", developed by Krupa and Szmolyan [14, which involves a change of variables to desingularize the invariant manifold.

We now proceed to describe the cartoon (the pieces of the solution in the singular limit) at the same time verifying the nondegeneracy hypotheses needed to carry out the perturbation.

4.1. Creating the dynamical problem. We start from (3.1.2)-3.1.3). It is convenient to introduce $V=\varepsilon \frac{d U}{d \xi}$, and $\theta=\xi-s_{\text {singular, }}$, and to treat $\xi$ as a state variable. This increases the dimension, but yields an autonomous system. Then the problem in the 
original self-similar variable (the slow time $\theta)$ is $\left(\right.$ with $\left.^{\prime}=d / d \theta\right)$

$$
\begin{aligned}
\varepsilon U^{\prime} & =V, \\
\varepsilon V^{\prime} & =(D F(U)-\xi I) V, \\
\xi^{\prime} & =1 .
\end{aligned}
$$

As written, this is clearly singular as $\varepsilon \rightarrow 0$. Replacing $\theta$ with $\tau$, where $\theta=\varepsilon \tau$, we will work completely in the fast time system $\left(\right.$ with $\left.{ }^{*}=d / d \tau\right)$

$$
\begin{aligned}
\dot{U} & =V, \\
\dot{V} & =(D F(U)-\xi I) V, \\
\dot{\xi} & =\varepsilon .
\end{aligned}
$$

We note that in this problem "slow" and "fast" do not correspond to "outer" and "inner". In fact, we will need an inner, faster time variable $\left(\eta=\tau / \varepsilon^{p}\right)$ to describe the inner solution, as done formally in the previous section.

The boundary conditions are

$$
(U, V, \xi)(-\infty)=\left(U_{L}, 0,-\infty\right), \quad(U, V, \xi)(+\infty)=\left(U_{R}, 0,+\infty\right) .
$$

By Fenichel [5], and as stated in Schecter [18], a system with normally hyperbolic manifolds of equilibria has perturbed normally hyperbolic invariant manifolds nearby. That is the case here: We may let $\varepsilon=0$ in (4.1.2), noting that 4.1.2) is now a regularly perturbed problem. With $\xi=$ const. for all solutions, the states $V=0$ are all equilibria, and they are the only equilibria.

Thus, the 3-dimensional space $S \equiv\{(U, V, \xi): V=0\}$ consists of equilibria when $\varepsilon=0$ and is an invariant subspace under (4.1.2) for every $\varepsilon$. In fact, the dynamics in this space, for $\varepsilon>0$, is simply linear motion in the increasing $\xi$ direction. Using the eigenvalues (2.2.2)-(2.2.3) we identify two subsets of $S$ : For $\delta>0$, we define 3-dimensional manifolds

$$
\begin{aligned}
& S_{0}=\left\{(U, V, \xi):\|U\| \leq \frac{1}{\delta}, \quad V=0, \quad \text { and } \quad \xi \leq \lambda_{1}(U)-\delta\right\}, \\
& S_{2}=\left\{(U, V, \xi):\|U\| \leq \frac{1}{\delta}, \quad V=0, \quad \text { and } \quad \lambda_{2}(U)+\delta \leq \xi\right\},
\end{aligned}
$$

which are normally hyperbolic since the lines $\xi=\lambda_{1}(U), \quad \xi=\lambda_{2}(U)$ are not included in the sets $S_{i}$. In fact, if we linearize (4.1.2) and set $V=0$, there are 3 eigenvalues of zero, with a full set of eigenvectors in the space of equilibria. The remaining eigenvalues, $-\xi+\lambda_{1}(U)$ and $-\xi+\lambda_{2}(U)$, are real and nonzero. In $S_{0}$, both are positive, so there is an unstable manifold of dimension 2 , and in $S_{2}$ a stable manifold of dimension 2 . The boundary value $\left(U_{L}, 0,-\infty\right)$ is an $\alpha$-limit of points in $S_{0}$, and $\left(U_{R}, 0,+\infty\right)$ an $\omega$ limit in $S_{2}$. For $\varepsilon>0$ and near 0, by Fenichel theory [5], the system (4.1.2) has normally hyperbolic invariant manifolds near each $S_{k}$. For a given $U_{L}$, we define the 1-dimensional invariant set

$$
S_{0}\left(U_{L}\right)=\left\{(U, V, \xi): U=U_{L}, \quad V=0, \quad \xi<\lambda_{1}\left(U_{L}\right)\right\} .
$$

By inspection, $S_{0}$ and $S_{0}\left(U_{L}\right)$ are invariant both for $\varepsilon=0$ and for $\varepsilon>0$, so the perturbed manifolds may be taken, in this case, to be the $S_{i}$ themselves. Thus the cartoon of the dynamics on this part of the flow is simply the constant state persisting as time, $\xi$, increases. This is the case both for the unperturbed system, with $\varepsilon=0$, and for $\varepsilon>0$. 
To obtain the desired behavior under perturbation, one also needs knowledge of the unstable manifolds of these invariant spaces. The manifold $S_{0}\left(U_{L}\right)$ possesses a 3 -dimensional unstable manifold $W_{\varepsilon}^{u}\left(S_{0}\left(U_{L}\right)\right)$, the perturbation of

$$
W_{0}^{u}\left(S_{0}\left(U_{L}\right)\right)=\left\{(U, V, \xi): U \in \Omega_{\xi}, \quad V=V(U), \quad \xi<\lambda_{1}\left(U_{L}\right)\right\},
$$

where $\Omega_{\xi}$ is an open subset of $U$-space that depends on $\xi$ and $U_{L}$. (The linearization of $W_{0}^{u}$ at a point in $S_{0}$ has a basis of eigenvectors, but we can ignore them for now, noting only that the projection of $W_{0}^{u}$ onto $U$-space contains a full neighborhood of $U_{L}$. The function $V(U)$ is determined by solving the system (4.1.2).) Similarly,

$$
S_{2}\left(U_{R}\right)=\left\{(U, V, \xi): U=U_{R}, \quad V=0, \quad \lambda_{2}\left(U_{R}\right)<\xi\right\}
$$

is a 1-dimensional set, which has a 3 -dimensional stable manifold, $W_{\varepsilon}^{s}\left(S_{2}\left(U_{R}\right)\right)$, the perturbation of

$$
W_{0}^{s}\left(S_{2}\left(U_{R}\right)\right)=\left\{(U, V, \xi): U \in \Omega_{\xi}, \quad V=V(U), \quad \lambda_{2}\left(U_{R}\right)<\xi\right\} .
$$

Since every trajectory in $W_{\varepsilon}^{u}\left(S_{0}\left(U_{L}\right)\right) \cap W_{\varepsilon}^{s}\left(S_{2}\left(U_{R}\right)\right)$ tends to $U_{R}$ as $\tau \rightarrow \infty$ and to $U_{L}$ as $\tau \rightarrow-\infty$, our objective is to show that these two 3 -dimensional manifolds intersect in 5-dimensional state space.

An alternative way of representing the solution is convenient. To focus attention on the shock layer, and specifically on the difficulties surrounding the Rankine-Hugoniot relation, which normally is derived from equations (3.1.14) and (3.1.16), we replace $V$ in (4.1.2) by

$$
W=-V+F(U)-\xi U .
$$

In addition, we treat $\varepsilon$ as a dynamical variable, for reasons that will become clear. Then we have the system

$$
\begin{aligned}
\dot{U} & =F(U)-\xi U-W, \\
\dot{W} & =-\varepsilon U, \\
\dot{\xi} & =\varepsilon, \\
\dot{\varepsilon} & =0 .
\end{aligned}
$$

Each subspace $\varepsilon=$ constant is invariant. The spaces $S_{0}$ and $S_{2}$ are now 4-dimensional, and still normally hyperbolic. We write them as

$$
\begin{array}{ll}
T_{0}=\{(U, W, \xi, \varepsilon): & \left.\|U\| \leq \frac{1}{\delta}, \quad W=F(U)-\xi U, \xi \leq \lambda_{1}(U)-\delta\right\}, \\
T_{2}=\{(U, W, \xi, \varepsilon): & \left.\|U\| \leq \frac{1}{\delta}, \quad W=F(U)-\xi U, \lambda_{2}(U)+\delta \leq \xi\right\} .
\end{array}
$$

The 1-dimensional sets $S_{0}\left(U_{L}\right)$ and $S_{2}\left(U_{R}\right)$ are now

$$
\begin{aligned}
& T_{0}^{\varepsilon}\left(U_{L}\right)=\left\{(U, W, \xi, \varepsilon): \quad U=U_{L}, W=F\left(U_{L}\right)-\xi U_{L}, \xi \leq \lambda_{1}\left(U_{L}\right)-\delta, \varepsilon \text { fixed }\right\}, \\
& T_{2}^{\varepsilon}\left(U_{R}\right)=\left\{(U, W, \xi, \varepsilon): U=U_{R}, W=F\left(U_{R}\right)-\xi U_{R}, \xi \geq \lambda_{2}\left(U_{R}\right)+\delta, \varepsilon \text { fixed }\right\},
\end{aligned}
$$

and we rewrite the 3 -dimensional unstable manifold $W_{\varepsilon}^{u}\left(S_{0}\left(U_{L}\right)\right)$ as

$$
W^{u}\left(T_{0}^{\varepsilon}\left(U_{L}\right)\right)=\left\{(U, W, \xi, \varepsilon): \quad U \in \Omega_{\xi}, \quad W=W(U), \xi<\lambda_{1}\left(U_{L}\right), \quad \varepsilon \text { fixed }\right\},
$$


where now $W(U)$ denotes the solution of (4.1.4) corresponding to $U$. Finally, the 3dimensional stable manifold $W_{\varepsilon}^{s}\left(S_{2}\left(U_{R}\right)\right)$ becomes a 3-dimensional space

$$
W^{s}\left(T_{2}^{\varepsilon}\left(U_{R}\right)\right)=\left\{(U, W, \xi, \varepsilon): U \in \Omega_{\xi}, W=W(U), \lambda_{2}\left(U_{R}\right)<\xi, \quad \varepsilon \text { fixed }\right\} .
$$

As with the previous coordinates, we look for a solution for fixed $\varepsilon>0$ that lies in the intersection of $W^{u}\left(T_{0}^{\varepsilon}\left(U_{L}\right)\right)$ and $W^{s}\left(T_{2}^{\varepsilon}\left(U_{R}\right)\right)$.

Now we write down an expression for the inner solution, motivated by the formal derivation given earlier. To fix ideas, we let $p=1$ (so $r=q=2$ ). It is possible that other choices would work, but we have not explored this. Then the scaling (3.1.4) introduces a new variable $Y=J(\varepsilon) U$, where

$$
J(x)=\left(\begin{array}{cc}
x & 0 \\
0 & x^{2}
\end{array}\right) .
$$

The flux function $F$ is invariant (up to a constant factor of $\varepsilon$ ) under this scaling, so the system, with a fast (or should we say 'faster'?) time variable $\eta=\tau / \varepsilon$ and ' $=d / d \eta$ is now

$$
\begin{aligned}
Y^{\prime} & =F(Y)-\varepsilon \xi Y-\varepsilon^{2} N(1, \varepsilon) W, \\
W^{\prime} & =-\varepsilon N(1,1 / \varepsilon) Y, \\
\xi^{\prime} & =\varepsilon^{2}, \\
\varepsilon^{\prime} & =0 .
\end{aligned}
$$

In (4.1.5), $N(x, y)=\operatorname{diag}(x, y)$. When $\varepsilon=0$, the equation for $Y$ decouples from the rest of the system, and is exactly (3.1.8). This is a small distinction from the case $\gamma=1$ considered in [11, although it appears to make no difference to the analysis. (In the case $\gamma=1$, the reduced system gives rise to a slightly different vector field.) Thus, (4.1.5) when $\varepsilon=0$ is

$$
\begin{aligned}
Y^{\prime} & =F(Y), \\
W^{\prime} & =-N(0,1) Y, \\
\xi^{\prime} & =0, \\
\varepsilon^{\prime} & =0 .
\end{aligned}
$$

The fact that $w_{1}$ and $w_{2}$ behave differently from each other is an indication that the asymmetry in the generalized Rankine-Hugoniot relation will enter into the analysis.

Now we see that $E=\{(Y, W, \xi, \varepsilon): Y=0, \varepsilon=0\}$ is a 3 -dimensional space consisting entirely of equilibria. If we linearize (4.1.6) at a point in $E$, we find that all 6 eigenvalues are zero. There is an eigenvector deficiency and, more important, $y_{2}=0$ for all eigenvectors. Thus, $E$ is not normally hyperbolic. This is exactly the situation found by Schecter in [18. It is resolved by "blowing up" the singularity at $Y=\varepsilon=0$.

4.2. The blow-up construction. Under the change of variables

$$
\begin{aligned}
Y & =J(\bar{r}) \bar{Y}, \\
\varepsilon & =\bar{r} \bar{\varepsilon},
\end{aligned}
$$

with $|\bar{Y}|^{2}+\bar{\varepsilon}^{2}=1$, the set $E$ becomes the set $\{\bar{r}=0\}$. This set is now 5-dimensional, in the 6 -dimensional $(\bar{Y}, \bar{\varepsilon}, \bar{r}, W, \xi)$-space $\mathbf{X}=S^{2} \times \mathbb{R}_{+} \times \mathbb{R}^{3}$. The system is also highly singular at $\{\bar{r}=0\}$, but becomes nonsingular upon division by $\bar{r}$. Thus, we can study 
the dynamics of the transformed system on $\mathbf{X}$. In terms of asymptotic structure, the change of variables (4.2.1) couples the growth of $U$ to the limit $\varepsilon \rightarrow 0$ in the fashion predicted by the formal asymptotics. Now the range of $\bar{Y}$ and $\bar{\varepsilon}$ is confined to the unit sphere, but the dynamics of these variables can be explored, and the result explains the connection between the bounded and unbounded parts of the singular shock. Analysis is made possible by the fact, suggested earlier, that we can find invariant sets of low dimension on $\mathbf{X}$ and can establish that they are normally hyperbolic. The homoclinic solution of Section 3.1 is one example, as we shall see. That solution provides the inner dynamics. In addition, connecting the inner solution to the limit points $U_{L}$ and $U_{R}$ can now be pursued, and this is the real innovation of the GSPT approach. (It is not clear, for example, that one could use GSPT without some prior knowledge or intuition about the asymptotics of the inner solution.) We will construct the invariant sets in $\mathbf{X}$ and establish their nondegeneracy.

The most straightforward way to describe the connection between the homoclinic orbit, which can be identified as the unique solution to (4.1.6) for which $w_{L 2}-w_{R 2}=k$ (the Rankine-Hugoniot deficit, from equation (3.1.19), and the states $U_{L}$ and $U_{R}$, which are limit points of the manifolds $W^{u}\left(T_{0}^{\varepsilon}\left(U_{L}\right)\right)$ and $W^{s}\left(T_{2}^{\varepsilon}\left(U_{R}\right)\right.$ ) (for $\varepsilon \geq 0$ ), is by defining intermediate points $q_{L}$ and $q_{R}$. In the coordinate system just introduced on $\mathbf{X}$, they are

$$
\begin{aligned}
& q_{L}=\left(a_{3} \sqrt{\bar{y}_{2}}, \bar{y}_{2}, 0,0, W_{L}, s\right), \\
& q_{R}=\left(a_{2} \sqrt{\bar{y}_{2}}, y_{2}, 0,0, W_{R}, s\right),
\end{aligned}
$$

where we have written the coordinates in the order $(\bar{Y}, \bar{\varepsilon}, \bar{r}, W, \xi) ; s$ is the speed of the singular shock, from (3.1.18); $a_{2}$ and $a_{3}$ are the two middle roots (in increasing order) of

$$
\frac{(2-\gamma)(5-3 \gamma)}{12} a^{4}-(2-\gamma) a^{2}+1=0
$$

and $\bar{y}_{2}$ is the positive root of $\bar{y}_{2}^{2}+a_{i}^{2} \bar{y}_{2}-1=0$ (so that $|\bar{Y}|^{2}+\bar{\varepsilon}^{2}=1$ ). Finally,

$$
W_{L}=F\left(U_{L}\right)-s U_{L}, \quad W_{R}=F\left(U_{R}\right)-s U_{R}
$$

we recall that $W=F\left(U_{i}\right)-\xi U_{i}(i=L, R)$ is the value of $W$ on the invariant sets $T_{0}\left(U_{L}\right)$ and $T_{2}\left(U_{R}\right)$, so $q_{L}$ and $q_{R}$ are specified by selecting the shock speed for $\xi$. These two points connect the inner and outer solutions. We shall show that there is a unique orbit connecting $U_{L}$ with $q_{L}$. Note that discussing such an orbit requires making the transition from the unscaled variables $(U, W, \xi, \varepsilon)$ to the coordinate system in $\mathbf{X}$. The connection between $q_{L}$ and $q_{R}$ is via the homoclinic orbit. Finally, $q_{R}$ connects to $U_{R}$ in the same manner as $U_{L}$ to $q_{L}$. Because the beginning and ending connections are similar, in the sequel we will look only at the first two steps. Figure 4.1 gives a sketch of the key parts of the solution. We begin with the definition of $q_{L}$ and $q_{R}$.

4.3. The first stage of the flow. From the description of the underlying planar system $U^{\prime}=F(U)$ or $Y^{\prime}=F(Y)$ and the sketch in Figure 3.1, it is intuitively clear that the flow trajectories are roughly parabolic. Specifically, if we consider (4.1.4) with $\varepsilon=0, \xi=s$ and $W=W_{L}=F\left(U_{L}\right)-s U_{L}$, then the equilibrium $U_{L}$ is a source. 
Proposition 4.1. The planar system $U^{\prime}=F(U)-s U-W_{L}$ contains a negatively invariant region to the right of $U_{L}$, bounded by the parabolic curves

$$
\begin{aligned}
& \phi_{1}\left(u_{1}\right)=A(\gamma)\left(u_{1}^{2}-u_{L 1}^{2}\right), \\
& \phi_{2}\left(u_{1}\right)=\frac{(3-\gamma)}{2}\left(u_{1}^{2}-u_{L 1}^{2}\right)-s\left(u_{1}-u_{L 1}\right),
\end{aligned}
$$

where $A(\gamma)$ depends on the value of $\gamma$; for example, if $1<\gamma<4 / 3$, then

$$
\begin{aligned}
& 1-\frac{\gamma}{2}-\frac{1}{2} \sqrt{(\gamma-1)\left(1-\frac{\gamma}{2}\right)}<A(\gamma)<\frac{1}{12}\left[11-6 \gamma+\sqrt{(\gamma-1)\left(1-\frac{\gamma}{2}\right)}\right] \\
&+\frac{1}{\sqrt{6}} \sqrt{\frac{\left[11-6 \gamma+\sqrt{(\gamma-1)\left(1-\frac{\gamma}{2}\right)}\right]^{2}}{24}-\frac{(2-\gamma)(5-3 \gamma)}{2}}
\end{aligned}
$$

Proof. A calculation of $u^{\prime}$ along the curves $\phi_{i}$, similar to Lemma 3.2 in [17, gives the result.

In particular, this means that trajectories within the open curvilinear wedge between these two curves all have $U_{L}$ as their $\alpha$-limits. A trajectory beginning near $U_{L}$ becomes unbounded, but the ratio $u_{1} / \sqrt{u_{2}}$ remains bounded. (In particular, the coordinate $u_{2}$ becomes positive after some time, so that defining this ratio makes sense.) This motivates introducing a new coordinate chart on $\mathbf{X}$, which we will call Chart 2, following Schecter's terminology in [18.

In terms of the coordinates $(\bar{Y}, \bar{\varepsilon}, \bar{r})$ (and, for reference, the scaled coordinates $(Y, \varepsilon)$ and the original coordinates $(U, \varepsilon))$, we define, on the portion of $\mathbf{X}$ where $\bar{y}_{2}$ is positive,

$$
\begin{aligned}
& a=\frac{\bar{y}_{1}}{\sqrt{\bar{y}_{2}}}=\frac{y_{1}}{\sqrt{y_{2}}}=\frac{u_{1}}{\sqrt{u_{2}}}, \\
& r^{2}=\bar{r}^{2} \bar{y}_{2}=y_{2}=\varepsilon^{2} u_{2}, \\
& b=\frac{\bar{\varepsilon}}{\sqrt{\bar{y}_{2}}}=\frac{\varepsilon}{\sqrt{y_{2}}}=\frac{1}{\sqrt{u_{2}}},
\end{aligned}
$$

and we again rescale the time variable, now to $r \eta$, which we will again call $\tau$, by an abuse of notation.

In these parabolic coordinates, the system (4.1.5) becomes

$$
\begin{aligned}
\frac{d a}{d \tau} & =(2-\gamma) a^{2}-1-\frac{(2-\gamma)(5-3 \gamma)}{12} a^{4}+\frac{b}{2}\left(-\xi a-2 b w_{1}+b^{2} a w_{2}\right) \\
\frac{d r}{d \tau} & =\frac{r}{6}\left(\frac{(2-\gamma)(5-3 \gamma)}{2} a^{3}-3 b \xi+3(\gamma-1) a-3 b^{3} w_{2}\right) \\
\frac{d w_{1}}{d \tau} & =-r a b \\
\frac{d w_{2}}{d \tau} & =-r \\
\frac{d \xi}{d \tau} & =r b^{2} \\
\frac{d b}{d \tau} & =-\frac{b}{6}\left(\frac{(2-\gamma)(5-3 \gamma)}{2} a^{3}-3 b \xi+3(\gamma-1) a-3 b^{3} w_{2}\right)
\end{aligned}
$$




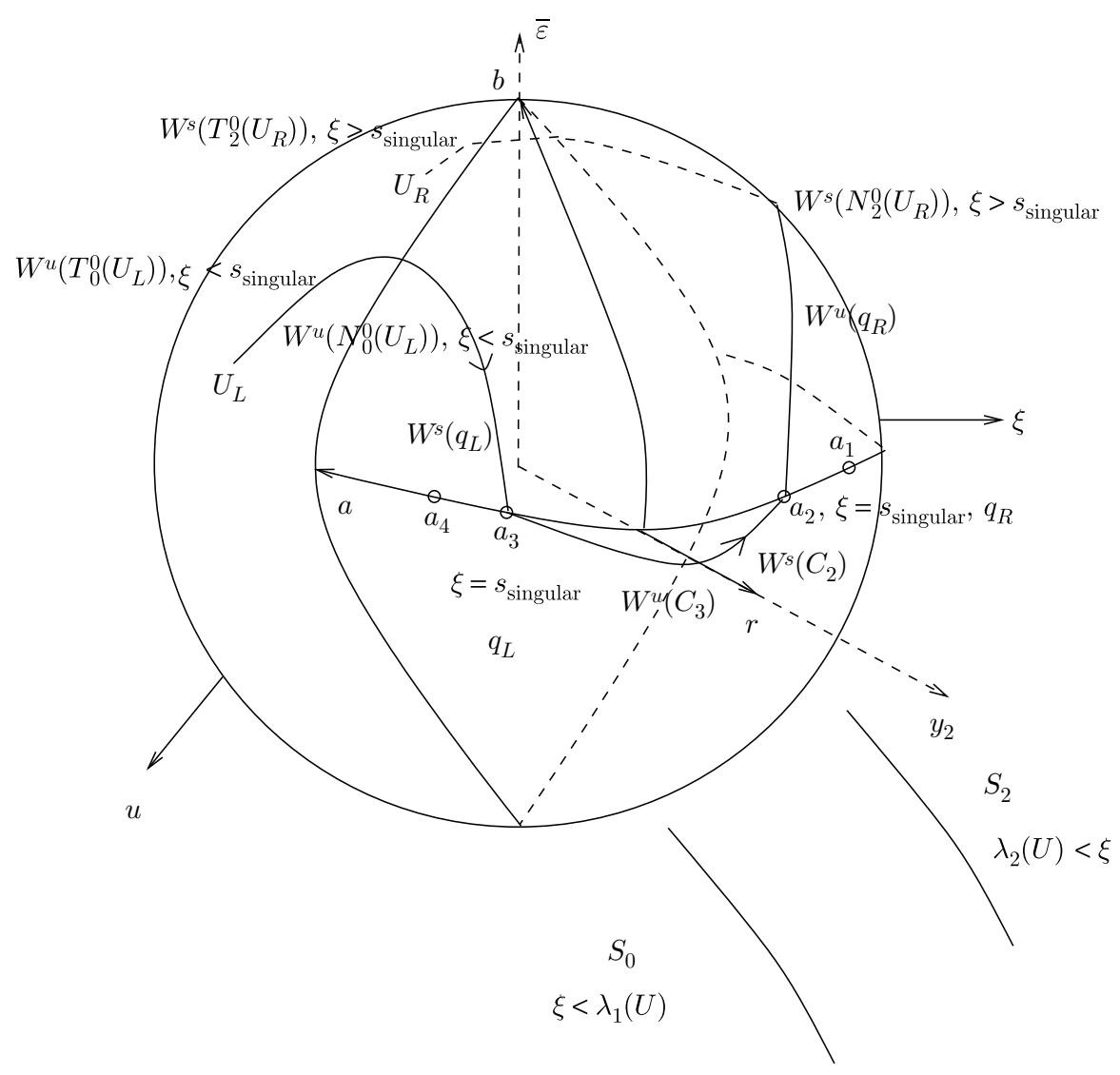

FIG. 4.1. Charts 1 and 2 .

The key point here is that the system (4.3.2) contains all the dynamics of the problem, scaled in a way that emphasizes a different part of the flow, namely the region where $U$ is growing to form the singular shock. In addition, this system also possesses an invariant manifold, which is normally hyperbolic, and we are able to prove existence of a solution to the Dafermos regularization, for small $\varepsilon$, by exhibiting a solution which is close to this invariant manifold during part of its trajectory.

As might be expected, we require $r=0$ (which corresponds to $\varepsilon=0$ ) to find invariant manifolds. In addition, in the region of interest we must set $b=0$, and then we have an equilibrium of (4.3.2) when $\frac{d a}{d \tau}=0$, that is, when $a$ is a root of the equation (4.2.4) introduced in the definitions of $q_{L}$ and $q_{R}$. The four roots of (4.2.4) are

$$
\begin{array}{ll}
a_{1}=-\sqrt{\frac{2}{(5-3 \gamma)}\left[3+\frac{\sqrt{3}}{\sqrt{2-\gamma}}\right],} & a_{2}=-\sqrt{\frac{2}{(5-3 \gamma)}\left[3-\frac{\sqrt{3}}{\sqrt{2-\gamma}}\right]}, \\
a_{3}=\sqrt{\frac{2}{(5-3 \gamma)}\left[3-\frac{\sqrt{3}}{\sqrt{2-\gamma}}\right],} & a_{4}=\sqrt{\frac{2}{(5-3 \gamma)}\left[3+\frac{\sqrt{3}}{\sqrt{2-\gamma}}\right] .}
\end{array}
$$


Using these roots, we define

$$
P_{j}=\left\{(a, r, W, \xi, b): a=a_{j}, r=0, b=0\right\} \quad \text { for } j=1,2,3,4 .
$$

Each of these sets is a 3-dimensional manifold of equilibria, corner equilibria in Schecter's definition 18. If we linearize (4.3.2) at $a=a_{j}, r=b=0$, we find a zero eigenvalue of multiplicity 3 , with 3 linearly independent eigenvectors lying in $P_{j}$. There are three additional eigenvalues,

$$
\begin{aligned}
& \lambda_{2}=\frac{2(2-\gamma)}{3} a_{j}\left[3-\frac{(5-3 \gamma)}{2} a_{j}^{2}\right], \\
& \lambda_{3}=\frac{(2-\gamma)(5-3 \gamma)}{12} a_{j}^{3}+\frac{(\gamma-1)}{2} a_{j}, \\
& \lambda_{4}=-\frac{(2-\gamma)(5-3 \gamma)}{12} a_{j}^{3}-\frac{(\gamma-1)}{2} a_{j},
\end{aligned}
$$

and since the corresponding eigenvectors, which are

$$
\begin{aligned}
& R_{2}=(1,0,0,0,0,0) \\
& R_{3}\left(a_{j}\right)=\left(0, \frac{(2-\gamma)(5-3 \gamma)}{12} a_{j}^{3}+\frac{(\gamma-1)}{2} a_{j}, 0,-1,0,0\right) \\
& R_{4}\left(a_{j}, \xi\right)=\left(\frac{\xi}{(7-3 \gamma)-\left(1-\frac{\gamma}{2}\right)(5-3 \gamma) a_{j}^{2}}, 0,0,0,0,1\right)
\end{aligned}
$$

respectively, are transversal to $P_{j}$, the $P_{j}$ are normally hyperbolic manifolds.

Only $P_{3}$ and $P_{2}$ play a role in what follows.

We fix a point $\left(a_{3}, 0, W_{0}, s_{\text {singular }}, 0\right)$ in $P_{3}$. Then $\lambda_{4}<0<\lambda_{2}, \lambda_{3}$, so the point has a 1-dimensional stable manifold tangent to $R_{4}\left(a_{3}, s_{\text {singular }}\right)$. Indeed, the stable manifold of any point with $r=b=0$ is contained in the 2-dimensional plane

$$
\left\{\left(a, r, w_{1}, w_{2}, \xi, b\right): r=0, W_{0}, \xi=s_{\text {singular }}\right\},
$$

which is invariant under the flow (4.3.2). Thus the stable manifold of $P_{3}$ is tangent to

$$
\left\{(a, r, W, \xi, b): r=0, b=\frac{\left[(7-3 \gamma)-\left(1-\frac{\gamma}{2}\right)(5-3 \gamma) a_{3}^{2}\right]}{\xi}\left(a-a_{3}\right)\right\}
$$

at $P_{3}$.

Since $\lambda_{2}$ and $\lambda_{3}$ are positive at points of $P_{3}$, each point has a 2-dimensional unstable manifold tangent to the plane spanned by $R_{2}$ and $R_{3}\left(a_{3}\right)$. (The same two eigenvalues, $\lambda_{2}$ and $\lambda_{3}$, are negative on $P_{2}$.) Thus $P_{3}$ has the 5 -dimensional unstable manifold

$$
W^{u}\left(P_{3}\right)=\left\{\left(a, r, w_{1}, w_{2}, \xi, b\right): b=0\right\} .
$$

The point $q_{L}$, identified earlier, is a particular point of $P_{3}$, with $W=W_{L}$ and $\xi=s_{\text {singular }}$. (In Chart 2 coordinates, $q_{L}=\left(a_{3}, 0, W_{L}, s_{\text {singular }}, 0\right)$.) Through the 1-dimensional stable manifold of $q_{L} \in P_{3}$, there is a unique connection backwards in time to $U_{L}$, and through the 2-dimensional unstable manifold, $q_{L}$ connects forward to the singular orbit. We state

Proposition 4.2. There is a unique orbit in the 2-dimensional invariant plane

$$
\left\{r=0, W=W_{L}, \xi=s_{\text {singular }}\right\}
$$


that connects $q_{L}$ as $\tau \rightarrow \infty$ with $U_{L}$ as $\tau \rightarrow-\infty$. Furthermore, in a neighborhood of $q_{L}$, we have $b>0$ along the orbit.

Proof. The proof is similar to the result of Schecter [18], with details motivated by Theorem 3.1 of [17. One can verify that, in one direction, the stable manifold of $q_{L}$ is in the interior of the negatively invariant region for $U_{L}$. The inequality for $b$ follows from examining the eigenvector tangent to the manifold at $q_{L}$. The coordinate system of Chart 2 is not suitable for describing the entire trajectory (this point was overlooked in [18, a minor oversight) because $u_{2}$ (or $y_{2}$ or $\bar{y}_{2}$ ) need not remain positive throughout the trajectory. Even if this were not a difficulty, the manifolds are described in different coordinate systems. The role of the Exchange Lemma is to guarantee, in the presence of normal hyperbolicity, which holds here, that one can exchange one coordinate system for the other.

A similar analysis shows a unique connection between $q_{R}$ and $U_{R}$, using properties of $P_{2}$.

To obtain the eventual result, which is a demonstration that connecting orbits exist in the neighborhood of these invariant manifolds, we need a related result. (It should be noted that the connection established between $q_{L}$ and $U_{L}$ does not solve the problem, since $q_{L}$ and $U_{L}$ are the $\omega$ - and $\alpha$-limits of a unique orbit, and thus are not themselves part of a longer connection between $U_{L}$ and $U_{R}$. Once we have found all the limiting pieces of the connections, we use the Corner Lemma to show that $U_{L}$ and $U_{R}$ can be connected when $\varepsilon>0$.) For this, we introduce 1-dimensional sets that contain $q_{L}$ and $q_{R}$ respectively. We define

$$
\begin{aligned}
& C_{3}=\left\{(a, r, W, \xi, b): a=a_{3}, r=0, W=F\left(U_{L}\right)-\xi U_{L}, \xi<\lambda_{1}\left(U_{L}\right), \quad b=0\right\}, \\
& C_{2}=\left\{(a, r, W, \xi, b): a=a_{2}, r=0, W=F\left(U_{R}\right)-\xi U_{R}, \lambda_{2}\left(U_{R}\right)<\xi, \quad b=0\right\},
\end{aligned}
$$

where we have now fixed the values of $W$ up to their variation with $\xi$, but have not fixed $\xi$ as we did to define $q_{i}$. The stable manifold of $C_{3}$ is 2 -dimensional; it is the union of the 1-dimensional stable manifolds of points that differ from $q_{L}$ only in the value of $\xi$. Since up to now we have not made use of the specific value of $\xi$ (beyond its relation to the eigenvalues of $d F\left(U_{L}\right)$ ), the results of Proposition 4.2 hold at each point of $C_{3}$, and we have (recalling that $T_{0}^{0}\left(U_{L}\right)$ is precisely the one-dimensional set in which $\xi$ is allowed to vary)

Proposition 4.3. In the coordinate system of Chart 2, the set $W^{u}\left(T_{0}^{0}\left(U_{L}\right)\right)$ takes the form

$$
W^{u}\left(N_{0}^{0}\left(U_{L}\right)\right)=\left\{\left(a_{\xi}(\tau), b_{\xi}(\tau), 0, W, \xi\right)\right\}
$$

where $W=F\left(U_{L}\right)-\xi U_{L}$ for a fixed $\xi<\lambda_{1}\left(U_{L}\right)$ and $\left(a_{\xi}, b_{\xi}\right)$, with $a=u_{1}(\tau) / \sqrt{u_{2}(\tau)}$ and $b=1 / \sqrt{u_{2}(\tau)}$ is the expression in Chart 2 coordinates of the solution of (4.3.2) with $\omega$-limit in $C_{3}$ for fixed $\xi$. The intersection of $W^{u}\left(N_{0}^{0}\left(U_{L}\right)\right)$ and $W^{s}\left(P_{3}\right)$ is an open subset $Q_{3}$ of $W^{s}\left(C_{3}\right)$, namely the points of $W^{s}\left(C_{3}\right)$ with $b>0$.

Proof. The conclusion of Proposition 4.2, which holds at each point of $C_{3}$, implies this result. The positivity of $b$ follows from the explicit scaling. 
The analogous result for $C_{2}$ and corresponding space $W^{s}\left(N_{2}^{0}\left(U_{R}\right)\right)$ are used to construct and analyse the second half of the orbit. For this purpose, we note that $P_{2}$ has a 5dimensional stable manifold

$$
W^{s}\left(P_{2}\right)=\{(a, r, W, \xi, b): b=0\} .
$$

4.4. The inner solution. We now turn our attention to connecting $q_{L}$ and $q_{R}$. Here, the lines $C_{3}$ and $C_{2}$ of equilibria, in $P_{3}$ and $P_{2}$, respectively, are useful. One may think of them as the places in which we will seek the connection between $q_{L}$ and $q_{R}$. In the coordinate system of Chart 2, they are segments of two parallel lines, through the points $q_{L}$ and $q_{R}$; those points lie in the subspace $\xi=s$, and $C_{3}$ and $C_{2}$ are orthogonal to that subspace. This part of the construction fails if we do not have the strict inequalities $s<\lambda_{1}\left(U_{L}\right)$ and $s>\lambda_{2}\left(U_{R}\right)$, because then one of $q_{L}$ or $q_{R}$ is an endpoint of the corresponding $C_{i}$ and we cannot verify Proposition 4.4, which we will need to apply the Corner Lemma.

The unstable manifold of $C_{3}$ and the stable manifold of $C_{2}$ both have dimension three, and we have a description of their tangent spaces at $C_{3}$ and $C_{2}$ respectively. They are spanned by the eigenvectors $R_{2}$ and $R_{3}$ of (4.3.3) and can be written

$$
\begin{aligned}
W^{u}\left(C_{3}\right)=\{(a, r, W, \xi, b) & : w_{1}=F_{1}\left(U_{L}\right)-\xi u_{L 1}, \\
r & =\left(\frac{(2-\gamma)(5-3 \gamma)}{12} a_{3}^{3}+\frac{(\gamma-1)}{2} a_{3}\right)\left[\frac{(2-\gamma)(5-3 \gamma)}{6} u_{L 1}^{3}\right. \\
& \left.+(\gamma-1) u_{L 1} u_{L 2}-\xi u_{L 2}-w_{2}\right], \\
& \left.\xi<\lambda_{1}\left(U_{L}\right), \quad b=0\right\}
\end{aligned}
$$

and

$$
\begin{aligned}
& W^{s}\left(C_{2}\right)=\left\{(a, r, W, \xi, b): w_{1}=F_{1}\left(U_{R}\right)-\xi u_{R 1},\right. \\
& r=\left(\frac{(2-\gamma)(5-3 \gamma)}{12} a_{2}^{3}+\frac{(\gamma-1)}{2} a_{2}\right)\left[\frac{(2-\gamma)(5-3 \gamma)}{6} u_{R 1}^{3}\right. \\
& \left.+(\gamma-1) u_{R 1} u_{R 2}-\xi u_{R 2}-w_{2}\right], \\
& \left.\lambda_{2}\left(U_{R}\right)<\xi, \quad b=0\right\} .
\end{aligned}
$$

We recall the definitions of $W_{L}$ and $W_{R}$, (4.2.5), and of $q_{L}$ and $q_{R}$ in the Chart 2 coordinate system

$$
q_{L}=\left(a_{3}, 0, W_{L}, s_{\text {singular }}, 0\right), \quad q_{R}=\left(a_{2}, 0, W_{R}, s_{\text {singular }}, 0\right) .
$$

By the assumptions of Theorem 3.1 $q_{L} \in C_{3}$ and $q_{R} \in C_{2}$. In addition, we note that using (3.1.22) $w_{L 1}=w_{R 1}$, and (3.1.23), $w_{R 2}=w_{L 2}-k<w_{L 2}$.

If we express $q_{L}$ and $q_{R}$ in $Y, \varepsilon$ coordinates, they are points in $E$ (the invariant set of equilibria of (4.1.6) $)$. Specifically, $q_{L}=\left(0, W_{L}, s_{\text {singular }}, 0\right)$ and $q_{R}=\left(0, W_{R}, s_{\text {singular }}, 0\right)$. Following the discussion of the homoclinic orbits in Section 3.1, there is a unique solution of (4.1.6) that connects the two points such that $y_{1}(0)=0, y_{2}(0)>0$ and $k=\int_{-\infty}^{\infty} y_{2}(\eta) d \eta>0$. Write the solution as

$$
\left(Y(\eta), W(\eta), s_{\text {singular }}, 0\right)
$$

with

$$
w_{1}(\eta)=w_{L 1}=w_{R 1}, \quad w_{2}(\eta)=w_{L 2}-\int_{-\infty}^{\eta} y_{2}(t) d t
$$


Since $y_{2} \geq 0$ and the ratio $y_{1} / \sqrt{y_{2}}$ is bounded on this orbit, it can be written in the coordinates of Chart $2,(a, r, W, \xi, b)$, in which it becomes

$$
q(\tau)=\left(a(\tau), r(\tau), W(\tau), s_{\text {singular }}, 0\right)
$$

with

$$
w_{1}=w_{L 1}, \quad w_{2}(\tau)=w_{L 2}-\int_{-\infty}^{\tau} r(\sigma) d \sigma
$$

Here $r( \pm \infty)=0, a(-\infty)=a_{3}, a(+\infty)=a_{2}$. We note that $q(-\infty)=q_{L}, q(+\infty)=q_{R}$; $r(\tau)$ is even and $a(\tau)$ is odd. Geometrically, $q(\tau)$ lies in the 3-dimensional subspace of $\mathbb{R}^{6}$ (in Chart 2 coordinates) with $w_{1}=w_{1 L}=w_{2 L}, \xi=s$ and $b=0$.

Proposition 4.4. The 3-dimensional manifolds $W^{u}\left(C_{3}\right)$ and $W^{s}\left(C_{2}\right)$ meet transversally along $q(\tau)$.

Proof. The tangent spaces to $W^{u}\left(C_{3}\right)$ and $W^{s}\left(C_{2}\right)$ are given by (4.4.1)-(4.4.2) and $q(\tau)$ by (4.4.3). Since $b=0$ we look only at the coordinates $(a, r, W, \xi)$. We linearize (4.3.2) along $q(\tau)$ to obtain

$$
\frac{d}{d \tau}\left(\begin{array}{c}
\bar{a} \\
\bar{r} \\
\bar{w}_{1} \\
\bar{w}_{2} \\
\bar{\xi}
\end{array}\right)=\left(\begin{array}{ccccc}
g_{1}(a) & 0 & 0 & 0 & 0 \\
r g_{2}(a) & g_{3}(a) & 0 & 0 & 0 \\
0 & 0 & 0 & 0 & 0 \\
0 & -1 & 0 & 0 & 0 \\
0 & 0 & 0 & 0 & 0
\end{array}\right)\left(\begin{array}{c}
\bar{a} \\
\bar{r} \\
\bar{w}_{1} \\
\bar{w}_{2} \\
\bar{\xi}
\end{array}\right),
$$

where

$$
\begin{aligned}
& g_{1}(a)=2(2-\gamma) a-\frac{(2-\gamma)(5-3 \gamma)}{3} a^{3}, \\
& g_{2}(a)=\frac{1}{4}(2-\gamma)(5-3 \gamma) a^{2}+\frac{(\gamma-1)}{2}, \\
& g_{3}(a)=\frac{1}{6}\left(\frac{(2-\gamma)(5-3 \gamma)}{2} a^{3}+3(\gamma-1) a\right) .
\end{aligned}
$$

We will verify that the orthogonal complements of $W^{u}\left(C_{3}\right)$ and $W^{s}\left(C_{2}\right)$, which are 2dimensional, are transversal, which will imply the same for the original spaces. For this, 
we use the adjoint equation,

$$
\frac{d}{d \tau}\left(\begin{array}{c}
\tilde{a} \\
\tilde{r} \\
\tilde{w}_{1} \\
\tilde{w}_{2} \\
\tilde{\xi}
\end{array}\right)=\left(\begin{array}{ccccc}
-g_{1}(a) & -r g_{2}(a) & 0 & 0 & 0 \\
0 & -g_{3}(a) & 0 & 1 & 0 \\
0 & 0 & 0 & 0 & 0 \\
0 & 0 & 0 & 0 & 0 \\
0 & 0 & 0 & 0 & 0
\end{array}\right)\left(\begin{array}{c}
\tilde{a} \\
\tilde{r} \\
\tilde{w_{1}} \\
\tilde{w_{2}} \\
\tilde{\xi}
\end{array}\right) .
$$

At $\tau=-\infty$ where $q=q_{L}$, and noting that $W^{\prime}=-U$, the tangent space of $W^{u}\left(C_{3}\right)$ is $T_{q_{L}} W^{u}\left(C_{3}\right)$, which is spanned by

$$
\begin{aligned}
& (1,0,0,0,0), \\
& \left(0, \frac{(2-\gamma)(5-3 \gamma)}{12} a_{3}^{3}+\frac{(\gamma-1)}{2} a_{3}, 0,-1,0\right), \\
& \left(0,0,-u_{L 1},-u_{L 2}, 1\right)
\end{aligned}
$$

and its complement is spanned by

$$
\begin{aligned}
& x_{1}=\left(0,0,1,0, u_{L 1}\right) \\
& x_{2}=\left(0,1,0, \frac{(2-\gamma)(5-3 \gamma)}{12} a_{3}^{3}+\frac{(\gamma-1)}{2} a_{3}, \frac{(2-\gamma)(5-3 \gamma)}{12} a_{3}^{3} u_{L 2}+\frac{(\gamma-1)}{2} a_{3} u_{L 2}\right) .
\end{aligned}
$$

Thus from (4.4.5) we find vectors $x_{1}(\tau), x_{2}(\tau)$ which span the complement of $T_{q(\tau)} W^{u}\left(C_{3}\right)$ along $q(\tau)$. Since $\tilde{w}_{1}, \tilde{w}_{2}$ and $\tilde{\xi}$ are constant, we seek the first two components of a spanning set

$$
\begin{aligned}
& x_{1}(\tau)=\left(*, *, 1,0, u_{L 1}\right), \\
& x_{2}(\tau)=\left(*, *, 0, \frac{(2-\gamma)(5-3 \gamma)}{12} a_{3}^{3}+\frac{(\gamma-1)}{2} a_{3}, \frac{(2-\gamma)(5-3 \gamma)}{12} a_{3}^{3} u_{L 2}+\frac{(\gamma-1)}{2} a_{3} u_{L 2}\right)
\end{aligned}
$$

(noting that the values at $\tau=-\infty$ were computed above). We integrate the first two equations of 4.4.5),

$$
\begin{aligned}
& \frac{d \tilde{a}}{d \tau}=-g_{1}(a) \tilde{a}-r g_{2}(a) \tilde{r} \\
& \frac{d \tilde{r}}{d \tau}=-\frac{1}{6}\left(\frac{(2-\gamma)(5-3 \gamma)}{2} a^{3}+3(\gamma-1) a\right) \tilde{r}+\tilde{w}_{2}
\end{aligned}
$$

obtaining

$$
\tilde{a}(\tau)=-\int_{-\infty}^{\tau} e^{-\int_{\sigma}^{\tau} g_{1}(a(\rho)) d \rho} r(\sigma) g_{2}(a(\sigma)) \tilde{r}(\sigma) d \sigma,
$$

since we have $\tilde{a}(-\infty)=0$. For $x_{1}(\tau)$ we have (using the computation at $\left.q_{L}\right) \tilde{w}_{2}(\tau)=0$ and $\tilde{r}(-\infty)=0$. Thus, from (4.4.5) we get $\tilde{r}=0$. Therefore

$$
x_{1}(\tau)=\left(\tilde{a}(\tau), 0,1,0, u_{L 1}\right),
$$

where $\tilde{a}(\tau)$ is given by (4.4.6). 
For $x_{2}(\tau)$ we have $\tilde{w}_{2}(\tau)=\frac{(2-\gamma)(5-3 \gamma)}{12} a_{3}^{3}+\frac{(\gamma-1)}{2} a_{3}$ and require $\tilde{r}(-\infty)=1$. Solving for $\tilde{r}$ gives

$$
\tilde{r}(\tau)=1+\int_{-\infty}^{\tau} e^{-\int_{\sigma}^{\tau} g_{3}(a(\rho)) d \rho}\left[-g_{3}(a(\sigma))+\tilde{w}_{2}\right] d \sigma .
$$

Therefore

$$
x_{2}(\tau)=\left(\tilde{a}(\tau), \tilde{r}(\tau), 0, \frac{(2-\gamma)(5-3 \gamma)}{12} a_{3}^{3}+\frac{(\gamma-1)}{2} a_{3}, \frac{(2-\gamma)(5-3 \gamma)}{12} a_{3}^{3} u_{L 2}+\frac{(\gamma-1)}{2} a_{3} u_{L 2}\right),
$$

where $\tilde{a}(\tau)$ and $\tilde{r}(\tau)$ are given by (4.4.6) and (4.4.7) respectively.

Similarly $T_{q_{R}} W^{s}\left(C_{2}\right)$ is spanned by

$$
\begin{aligned}
& (1,0,0,0,0), \\
& \left(0, \frac{(2-\gamma)(5-3 \gamma)}{12} a_{2}^{3}+\frac{(\gamma-1)}{2} a_{2}, 0,-1,0\right), \\
& \left(0,0,-u_{R 1},-u_{R 2}, 1\right)
\end{aligned}
$$

and its complement by

$$
\begin{aligned}
& \theta_{1}=\left(0,0,1,0, u_{R 1}\right), \\
& \theta_{2}=\left(0,1,0, \frac{(2-\gamma)(5-3 \gamma)}{12} a_{2}^{3}+\frac{(\gamma-1)}{2} a_{2}, \frac{(2-\gamma)(5-3 \gamma)}{12} a_{2}^{3} u_{R 2}+\frac{(\gamma-1)}{2} a_{2} u_{R 2}\right),
\end{aligned}
$$

which identifies the tangent spaces to $W^{s}\left(C_{2}\right)$ along $q$ at $\tau=+\infty$. Integrating in the same way as for $x_{1}$ and $x_{2}$ we construct a spanning set $\theta_{1}(\tau), \theta_{2}(\tau)$ for the complement of $T_{q(\tau)} W^{s}\left(C_{2}\right)$. We find

$$
\begin{aligned}
& \theta_{1}(\tau)=\left(\hat{a}(\tau), 0,1,0, u_{R 1}\right), \\
& \theta_{2}(\tau)=\left(\hat{a}(\tau), \hat{r}(\tau), 0, \frac{(2-\gamma)(5-3 \gamma)}{12} a_{2}^{3}+\frac{(\gamma-1)}{2} a_{2}, \frac{(2-\gamma)(5-3 \gamma)}{12} a_{2}^{3} u_{R 2}+\frac{(\gamma-1)}{2} a_{2} u_{R 2}\right),
\end{aligned}
$$

where

$$
\begin{aligned}
\hat{a}(\tau) & =-\int_{\infty}^{\tau} e^{-\int_{\sigma}^{\tau} g_{1}(a(\rho)) d \rho} r(\sigma) g_{2}(a(\sigma)) \hat{r}(\sigma) d \sigma, \\
\hat{w}_{2}(\tau) & =\frac{(2-\gamma)(5-3 \gamma)}{12} a_{2}^{3}+\frac{(\gamma-1)}{2} a_{2}, \\
\hat{r}(\tau) & =1-\int_{\tau}^{\infty} e^{-\int_{\sigma}^{\tau} g_{3}(a(\rho)) d \rho}\left[-g_{3}(a(\sigma))+\hat{w}_{2}\right] d \sigma .
\end{aligned}
$$

Finally, to establish transversality, it is sufficient to check that $x_{i}$ and $\theta_{i}$ form a linearly independent set at a single point, say $\tau=0$. We suppress the first component and compute

$$
\begin{aligned}
\operatorname{det}\left(\begin{array}{cccc}
0 & 1 & 0 & u_{L 1} \\
\tilde{r}(0) & 0 & \tilde{w}_{2}(0) & \frac{(2-\gamma)(5-3 \gamma)}{12} a_{3}^{3} u_{L 2}+\frac{(\gamma-1)}{2} a_{3} u_{L 2} \\
0 & 1 & 0 & u_{R 1} \\
\hat{r}(0) & 0 & \hat{w}_{2}(0) & \frac{(2-\gamma)(5-3 \gamma)}{12} a_{2}^{3} u_{R 2}+\frac{(\gamma-1)}{2} a_{2} u_{R 2}
\end{array}\right) \\
=-(\tilde{r}(0)+\hat{r}(0))\left(u_{R 1}-u_{L 1}\right)\left[\frac{(2-\gamma)(5-3 \gamma)}{12} a_{3}^{3}+\frac{(\gamma-1)}{2} a_{3}\right]
\end{aligned}
$$


(since $a_{2}=-a_{3}$ ). Note that $u_{L 1} \neq u_{R 1}$. Noting that $a(\tau)$ in (4.4.3) is odd, if we define

$$
f(\sigma)=e^{-\int_{\sigma}^{0} \frac{1}{6}\left(\frac{(2-\gamma)(5-3 \gamma)}{2} a(\rho)^{3}+3(\gamma-1) a(\rho)\right) d \rho},
$$

we can see that $f$ is even. We calculate

$$
\begin{aligned}
\tilde{r}(0)+\hat{r}(0)=2 & -\int_{-\infty}^{\infty} f(\sigma)\left[\frac{(2-\gamma)(5-3 \gamma)}{12} a(\sigma)^{3}+\frac{(\gamma-1)}{2} a(\sigma)\right] d \sigma \\
& +\int_{-\infty}^{0} f(\sigma)\left[\frac{(2-\gamma)(5-3 \gamma)}{12} a_{3}^{3}+\frac{(\gamma-1)}{2} a_{3}\right] d \sigma \\
& -\int_{0}^{\infty} f(\sigma)\left[\frac{(2-\gamma)(5-3 \gamma)}{12} a_{3}^{3}+\frac{(\gamma-1)}{2} a_{3}\right] d \sigma \\
= & 2 .
\end{aligned}
$$

(The first integral, of an odd function, is zero, and the second and third integrals cancel each other out.) The determinant is not zero; therefore the four vectors are linearly independent. This completes the proof.

This result will allow us to match $W^{u}\left(C_{3}\right)$ with $W^{s}\left(C_{2}\right)$ in the proof of Theorem 3.1 .

4.5. Completion of the result. The components of the solution of the problem are now in place. Three particular orbits have been constructed, each corresponding to the limit $\varepsilon=0$ : $A_{1}$ joining $U_{L}$ to $q_{L}, A_{2}$ joining $q_{L}$ to $q_{R}$, and $A_{3}$ joining $q_{R}$ to $U_{R}$. To show that a solution exists for $\varepsilon>0$, that will actually connect $U_{L}$ and $U_{R}$ via a solution of the equation, we need to show that there is a solution, with $\varepsilon>0$, that is close to the union of these three orbits. The technique is to show that a solution close to $A_{1}$, in $W^{u}\left(T_{0}^{\varepsilon}\left(U_{L}\right)\right)$, will enter $W^{u}\left(C_{3}\right)$, and similarly to match $W^{s}\left(C_{2}\right)$ with $W^{s}\left(T_{2}^{\varepsilon}\left(U_{R}\right)\right)$. We do this by verifying the conditions of the Corner Lemma (Theorem 5.1 of Schecter [18]).

Proposition 4.5. In the coordinate system of Chart 2, the sets $W^{u}\left(T_{0}^{\varepsilon}\left(U_{L}\right)\right)$ and $W^{s}\left(T_{2}^{\varepsilon}\left(U_{R}\right)\right)$ will be denoted by $W^{u}\left(N_{0}^{\varepsilon}\left(U_{L}\right)\right)$ and $W^{s}\left(N_{2}^{\varepsilon}\left(U_{R}\right)\right)$, respectively.

The 4-dimensional set $W^{u}\left(N_{0}\left(U_{L}\right)\right)=\bigcup_{0 \leq \varepsilon \leq \varepsilon_{0}} W^{u}\left(N_{0}^{\varepsilon}\left(U_{L}\right)\right)$ is transverse to $W^{s}\left(P_{3}\right)$ along $Q_{3}$, and $W^{s}\left(N_{2}\left(U_{R}\right)\right)=\bigcup_{0 \leq \varepsilon \leq \varepsilon_{0}} W^{s}\left(N_{2}^{\varepsilon}\left(U_{R}\right)\right)$ is transverse to $W^{u}\left(P_{2}\right)$ along $Q_{2}$.

Proof. When we calculate $W^{u}\left(N_{0}\left(U_{L}\right)\right)$ at $Q_{3}$ in the coordinate system of Chart 2, we find that the tangent space to $W^{u}\left(N_{0}\left(U_{L}\right)\right)$ is spanned by

$$
\begin{aligned}
& (1,0,0,0,0,0), \\
& (0,0,0,0,0,1), \\
& \left(0,0,-u_{L 1},-u_{L 2}, 1,0\right), \\
& (0,1,0,0,0,0) .
\end{aligned}
$$

The tangent space to $W^{s}\left(P_{3}\right)$ at the same point is spanned by

$$
\begin{aligned}
& (0,0,1,0,0,0), \\
& (0,0,0,1,0,0) .
\end{aligned}
$$

These six vectors are linearly independent; therefore transversality follows. 


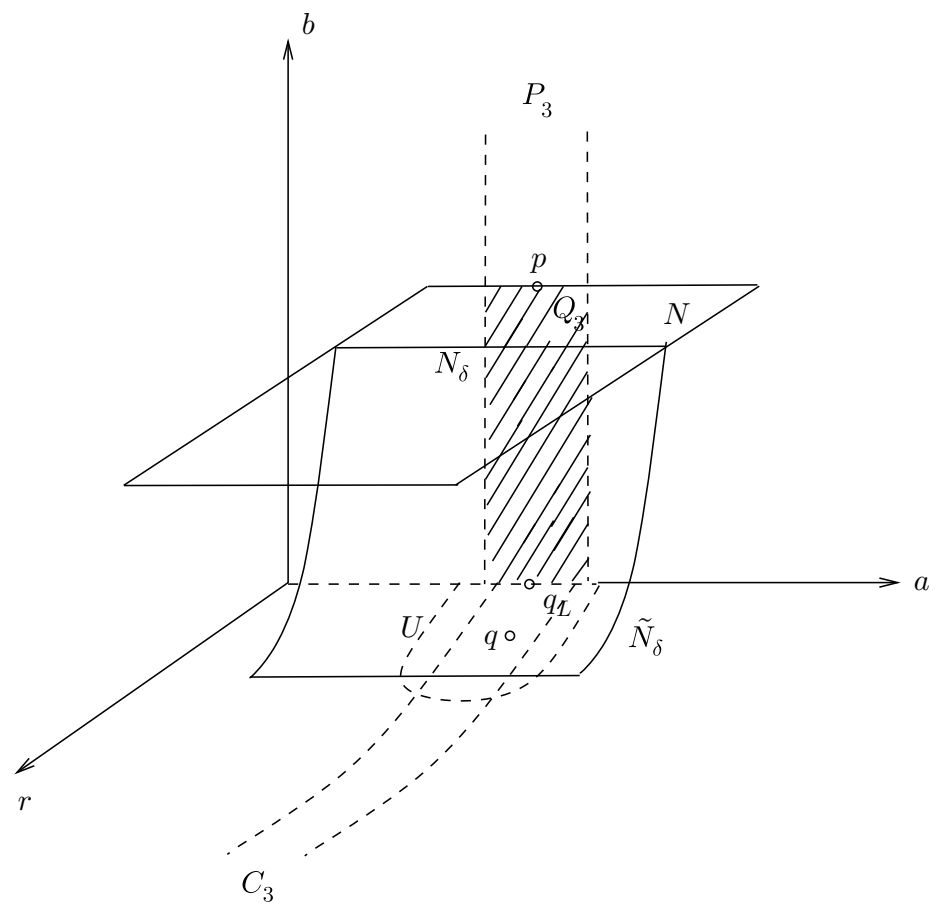

Fig. 4.2. Corner Lemma.

Proof of Theorem 3.1. Proposition 4.5 establishes the hypotheses of the Corner Lemma [18. As Schecter showed in [18, we have the 1-dimensional space

$$
W^{s}\left(q_{L}\right)=\left\{(a, r, W, \xi, b): r=0, \quad W=W_{L}, \xi=s_{\text {singular }}, \quad b=b(a)\right\},
$$

where, from the expression for the tangent space of $W^{s}\left(P_{3}\right)$, (4.3.4),

$$
b=\frac{\left[(7-3 \gamma)-\left(1-\frac{\gamma}{2}\right)(5-3 \gamma) a_{3}^{2}\right]}{s_{\text {singular }}}\left(a-a_{3}\right)+\cdots
$$

near $a_{3}$. See Figure 4.2. We let $p \in W^{s}\left(q_{L}\right) \backslash\left\{q_{L}\right\}$, let $N$ be a 3 -dimensional slice of $W^{u}\left(N_{0}\left(U_{L}\right)\right)$ transverse to the vector field and to $W^{s}\left(P_{3}\right)$ at the point $p$; let $N_{\delta}=$ $N \cap\{r=\delta\}$, a 2-dimensional manifold; let $q$ be in $W^{u}\left(C_{3}\right)$ with positive $r$ coordinate, and let $U$ be a small neighborhood of $q$.

Then under the flow, $N_{\delta}$ becomes a 3-dimensional manifold $\tilde{N}_{\delta}$ (like $W^{u}\left(N_{0}^{\delta}\left(U_{L}\right)\right)$ ) that passes near $q$. By the Corner Lemma,

$$
\text { as } \delta \rightarrow 0, \tilde{N}_{\delta} \cap U \rightarrow W^{u}\left(C_{3}\right) \cap U \text { in the } C^{1} \text { topology. }
$$

With the lemma we make the final match for the solution since $W^{u}\left(N_{0}^{\varepsilon}\left(U_{L}\right)\right)$ passes $q_{L}$ and arrives near $q(-T)$ for $T>0$. Similarly $W^{s}\left(N_{2}^{\varepsilon}\left(U_{R}\right)\right)$ passes $q_{R}$ in backward time and arrives near $q(T)$, where $q(\cdot)$ is given by (4.4.3). We then have a solution connecting $U_{L}$ and $U_{R}$. As $\varepsilon \rightarrow 0$, this solution is unbounded. This completes the proof of Theorem 3.1 . 
Acknowledgments. We are grateful to Steve Schecter for many helpful conversations about the implementation of GSPT, and to Peter Szmolyan for explaining some of the background. We offer many thanks to the organizers of the 2011 conference in honor of Constantine Dafermos for the opportunity to present some of this work and to contribute to the volume commemorating the event.

\section{REFERENCES}

[1] A. Bressan, Hyperbolic systems of Conservation Laws: The One-Dimensional Cauchy Problem, Oxford University Press, Oxford, 2000. MR1816648 (2002d:35002)

[2] C. M. Dafermos, Solution of the Riemann problem for a class of hyperbolic systems of conservation laws by the viscosity method. Arch. Rational Mech. Anal. 52 (1973), 1-9. MR0340837 (49:5587)

[3] C. M. Dafermos and R. J. DiPerna, The Riemann problem for certain classes of hyperbolic systems of conservation laws. J. Differential Equations 20 (1976), 90-114. MR0404871 (53:8671)

[4] B. Deng, Homoclinic bifurcations with nonhyperbolic equilibria. SIAM J. Math. Anal. 21 (1990), 693-719. MR1046796 (91g:58200)

[5] N. Fenichel, Geometric singular perturbation theory for ordinary differential equations, J. Differential Equations 31 (1979), 53-98. MR524817 (80m:58032)

[6] C. K. R. T. Jones, Geometric singular perturbation theory. Dynamical systems (Montecatini Terme, 1994), Lecture Notes in Mathematics, Vol. 1609, Springer, Berlin, 1995, pp. 44-118. MR1374108 (97e:34105)

[7] C. K. R. T. Jones and T. Kaper, A primer on the exchange lemma for fast-slow systems. Multipletime-scale dynamical systems (Minneapolis, MN, 1997) IMA Vol. Math. Appl. 122 (2001), 85-132. MR.1846573 (2002g:37022)

[8] C. K. R. T. Jones and N. Kopell, Tracking invariant manifolds with differential forms in singularly perturbed systems. J. Differential Equations 108 (1994), 64-88. MR.1268351(95c:34085)

[9] B. L. Keyfitz, Conservation laws, delta shocks and singular shocks. In Nonlinear Theory of Generalized Functions (eds. M. Grosser et al.), Chapman \& Hall/CRC Press, Boca Raton, 1999, pp. 99-111. MR.1699874

[10] B. L. Keyfitz, A new look at singular shocks. Confluentes Matematici, to appear, 2012.

[11] B. L. Keyfitz and H. C. Kranzer, A viscosity approximation to a system of conservation laws with no classical Riemann solution. In Nonlinear Hyperbolic Problems (Bordeaux, 1998), (eds. C. Carasso et al.), Lecture Notes in Mathematics, Vol. 1402, Springer, Berlin, 1989, pp. 185-197. MR.1033283 (90k:35168)

[12] B. L. Keyfitz and H. C. Kranzer, Spaces of weighted measures for conservation laws with singular shock solutions, J. Differential Equations, 118 (1995), 420-451. MR1330835 (96b:35138)

[13] H. C. Kranzer and B. L. Keyfitz, A strictly hyperbolic system of conservation laws admitting singular shocks. In Nonlinear Evolution Equations that Change Type (eds. B. L. Keyfitz and M. Shearer), Springer, New York, 1990, pp. 107-125. MR1074189 (92g:35133)

[14] M. Krupa and P. Szmolyan, Extending geometric singular perturbation theory to nonhyperbolic points - fold and canard points in two dimensions, SIAM J. Math. Anal., 33 (2001), 286-314. MR.1857972 (2002g:34117)

[15] M. Mazzotti, Non-classical composition fronts in nonlinear chromatography - Delta-shock, Indust. \& Eng. Chem. Res., 48 (2009), 7733-7752.

[16] M. Mazzotti, A. Tarafder, J. Cornel, F. Gritti and G. Guiochon, Experimental evidence of a deltashock in nonlinear chromatography, J. Chromatography. A, 1217(13) (2010), 2002-2012.

[17] D. G. Schaeffer, S. Schecter and M. Shearer, Nonstrictly hyperbolic conservation laws with a parabolic line, J. Differential Equations 103 (1993), 94-126. MR1218740 (94d:35102)

[18] S. Schecter, Existence of Dafermos profiles for singular shocks, J. Differential Equations 205 (2004), 185-210. MR2094383 (2005k:35269)

[19] S. Schecter and P. Szmolyan, Composite waves in the Dafermos regularization. J. Dynamics and Differential Equations 16 (2004), 847-867. MR2109169 (2005h:35234)

[20] M. Sever, Distribution solutions of nonlinear systems of conservation laws, Memoirs of the AMS, 889 (2007), 1-163. MR2355635 (2008k:35313)

[21] M. Sever, Large-data solution of a model system for singular shocks, J. of Hyperbolic Differential Equations 7 (2010), 775-840. MR2746206 (2012c:35270) 